\title{
Interactive effects of multiple vernalization (Vrn-1)- and photoperiod (Ppd-1)-related genes on the growth habit of bread wheat and their association with heading and flowering time
}

Shulin Chen ${ }^{1}$, Junsen Wang ${ }^{1}$, Genwang Deng ${ }^{2}$, Long Chen ${ }^{3}$, Xiyong Cheng ${ }^{1}$, Haixia Xu ${ }^{1}$ and Kehui Zhan ${ }^{*}$

\begin{abstract}
Background: The precise identification of Winterness/Springness (growth habit) for bread wheat, which is determined by genes involved in vernalization and photoperiod, will contribute to the effective utilization of bread wheat varieties. Here, 198 varieties from the Yellow and Huai wheat production region (YHW) in China were collected to identify their vernalization ( $\mathrm{r} r \mathrm{n}-1)$ and photoperiod (Ppd-1) gene composition via a series of functional markers and their association with vernalization and photoperiod requirements at three locations during two years of experiments. The growth habits were measured during the spring sowing season.

Results: The results showed that the semi-winter varieties (grades1-4) were most prevalent in the population. The relative effects of single $V r n$ alleles on the growth period, such as heading date (HD) and/or flowering date (FD), were as follows: $V r n-B 1 b>V r n-B 1 a>V r n-D 1 b>V r n-D 1 a>v r n-D 1=v r n-B 1$. The interactive effects of Vrn-B1 and Vrn$\mathrm{D} 1 \mathrm{on} \mathrm{HD}$ and FD were identical to those of Vrn-B1b. Approximately $35.3 \%$ of the cultivars carried Ppd-B1a (photoperiod-insensitive) and exhibited the earliest HD and FD. The Ppd-Dla-insensitive allele (Hapl II) was carried by just $0.5 \%$ of the varieties; however, the other two sensitive alleles were present at a higher frequency, and their effects were slightly weaker than those of Ppd-B1a. In addition, strong interactive effects between Ppd-B1 and Ppd-D1 were detected. In terms of mean values among various genotypes, the effects followed the order of Vrn-1>Ppd-1.

Conclusions: According to the results of ANOVA and least significant range (LSR) tests, we can conclude that Vrn-1 rather than Ppd-1 played a major role in controlling vernalization and photoperiod responses in this region. This research will be helpful for precisely characterizing and evaluating the HD, FD and even growth habit of varieties in the YHW at molecular levels.
\end{abstract}

Keywords: Winter wheat, The yellow and Huai wheat production region (YHW), Growth habit, Growth period, Vernalization, Photoperiod

\footnotetext{
*Correspondence: kh486@163.com

${ }^{1}$ College of Agronomy, Henan Agricultural University/Collaborative

Innovation Center of Henan Grain Crops, Zhengzhou 450002, China

Full list of author information is available at the end of the article
}

(c) The Author(s). 2018 Open Access This article is distributed under the terms of the Creative Commons Attribution 4.0 International License (http://creativecommons.org/licenses/by/4.0/), which permits unrestricted use, distribution, and reproduction in any medium, provided you give appropriate credit to the original author(s) and the source, provide a link to the Creative Commons license, and indicate if changes were made. The Creative Commons Public Domain Dedication waiver (http://creativecommons.org/publicdomain/zero/1.0/) applies to the data made available in this article, unless otherwise stated. 


\section{Background}

Because of the ease of determining its optimum time for flowering and maturation, bread wheat (Triticum aestivum L., AABBDD, $2 n=42$ ) is cultivated worldwide. Day length and low temperature act as environmental cues affecting the time to heading and flowering. The ability to perceive and respond to these signals is controlled by molecular pathways that regulate early growth habits in response to abiotic stress ( $\mathrm{Vrn}$ alleles) and photoperiod (Ppd alleles) [1, 2].

In the past, the characterization of the growth habits of winter wheat in China, especially in the Yellow and Huai wheat production region, which is the largest winter wheat production region, relied on field evaluations or in-house artificial identification [3, 4]. However, these identification procedures are tedious and costly, and thus, the practicality of field evaluation and in-house artificial identification methods is limited. Furthermore, inconsistencies between breeders' descriptions and government registrations sometimes occur due to inexact phenotypic identification methods. In contrast, molecular identification methods are relatively credible, but more novel types of vernalization alleles must be surveyed, and their interactive effects among each other remain unclear.

Indeed, the molecular basis for flowering time regulation has been extensively studied in wheat and other crops [5]. In hexaploid wheat, vernalization requirements are controlled by three major orthologous Vrnalleles$V r n-A 1, V r n-B 1$, and Vrn-D1-which have been mapped onto the long arm of chromosomes $5 \mathrm{~A}, 5 \mathrm{~B}$, and $5 \mathrm{D}$, respectively [6-8]. Each of these loci encodes a MADS-box transcription factor orthologous to AP1 in Arabidopsis, which is reported to be involved in floral meristem development during the transition from the vegetative phase to the reproductive phase [8]. The $V R N-1$ gene is dominant for the spring growth habit, and it is upregulated by vernalization in winter lines $[9,10]$. A homologue of the Arabidopsis FT gene, the Vrn-3 gene, has been mapped to the short arm of chromosome 7 in wheat; this gene upregulated the Vrn-1 genes and thus accelerated heading and flowering indirectly [11].

The emergence of dominant alleles at the Vrn-A1 locus is a result of insertions and deletions within the promoter or a deletion within intron 1, which have been designated $V r n-A 1 a, V r n-A 1 b$, and $V r n-A 1 c$, respectively $[9,10,12,13]$. Spring growth habits can also be attributed to deletions at the Vrn-B1 and Vrn-D1 loci, which have been classified as insensitive vernalization types, and have been designated Vrn-B1a [10, 14, 15], Vrn-D1a, and $V r n-D 1 b$ [16]. The $V r n-B 1 c$ (novel) allele, which is due to the deletion of $0.8 \mathrm{~kb}$ and the duplication of 0.4 $\mathrm{kb}$ within intron 1, differs from Vrn-B1a [17, 18]. Another spring allele, $V r n-B 1 b$, has also been described; this allele contains two deletions in the promoter region and is present in spring variety 'Alpowa' [14]. The various vernalization requirements of the $\mathrm{Vrn}-1$ alleles or combinations can result in variations in flowering time and spring growth habit [19]. In wheat and other temperate grasses, VRN1 is also expressed in the leaves, where it acts as a repressor of VRN2 [20, 21]. The detailed pathway of the vernalization genes involved in controlling wheat flowering was reviewed by Chen and Dubcovsky (2012) [20].

Photoperiod response is another vital factor affecting flowering time under long-day conditions. For wheat, photoperiod insensitivity $(P p d-1 a)$ is widespread and especially prevalent in regions where crops grow during short days or when the crops mature before the onset of high summer temperatures [22]. Three semi-dominant orthologous Ppd-1 loci-Ppd-A1, Ppd-B1, and Ppd-D1have been mapped onto the short arm of chromosomes $2 \mathrm{~A}, 2 \mathrm{~B}$, and $2 \mathrm{D}$, respectively $[23,24]$; these loci are all members of the Pseudo-Response Regulator (PRR) gene family, which is orthologous to the $\mathrm{Ppd}-\mathrm{H} 1$ gene family in barley [25]. A series of diagnostic markers have been used to efficiently screen for several variants $[10,13,16,24,26]$.

The Yellow and Huai valley wheat production region (YHW) covers $45 \%$ of China's total cultivation area but contributes $60-70 \%$ of the country's wheat production. Varieties that flower and mature early are helpful in sustaining China's double-harvest cropping system. In this study, we collected and identified a total of 198 popular varieties, elite lines, and landraces from China to (i) accurately identify the growth habits of the varieties via the field spring sowing method and evaluate their association with heading date (HD; growth period) and flowering date (FD; growth period) at three locations within the YHW during a two-year period (Zhengzhou, Zhumadian and Shangqiu in 2014 and 2015); (ii) use diagnostic molecular markers to determine the main allelic frequencies of $V r n-1$ and $P p d-1$; and (iii) specifically determine the interactive effects between $\mathrm{Vrn}-1$ and $P p d-1$ allelic combinations on heading and flowering times. This study contributes knowledge concerning the effective selection of various types of growth habits of varieties and will be of service to the selection of early-maturation cultivars at the molecular level.

\section{Results}

Semi-winter varieties were predominant in the YHW according to the field spring sowing method.

The results of the two-year growth habits were very similar, and the order ranks recorded in 2015 strongly correlated with those recorded in 2016 (Pearson coefficient $=0.96)$. In general, the ranks of two accessions (Xinong979 and Yumai47) were inconsistent between 
years, but the discrepancies were only 1-2 grades. Our method separated 10 accessions (Yannong19, Beijing841, etc.) into winterness (grade 0) in 2015 and 2016, which accounted for $5.05 \%$. One hundred and forty-seven accessions (74.24\%) were identified as semi-winter types in 2015, and 145 (73.23\%) were identified in 2016. In contrast, $41(20.71 \%)$ and $43(21.72 \%)$ grade 5 accessions belonged to the spring type in 2015 and 2016. Overall, the semi-winter varieties (grades1-4) were predominant in the YHW (Table 1; Fig. 1).

In comparison with the data on regional trials (registered, Table 1), our data showed that the consistency was $89.39 \%$ when the accessions were divided into winter and spring groups, although 21 varieties need to be re-examined. In detail, of the varieties identified in the winter group, 10 varieties, including Huangming116, Lankaoaizao8, Yumai4, Taikong6, Zhengmai101, Zhongchuang805, Zhoumai23, Zhongyanmai 0708, Huaimai 19 and Xiaoyan 22, qualified as week spring (spring) type according to the registered results. The 11 spring growth habit accessions, which included Jihan2, Hengguan35, Zhengyou6, Luohan6, Luomai23, Pu2056, Shanyou225, Xinong9871, Yunong416, Zhoumai26 and Huarui 00712, were misclassified as accessions having winter (or semi-winter) growth habits. The reason is that these varieties were registered ten years ago, and winter-spring identification was not evaluated during registration tests at that time.

\section{Growth habits were highly associated with growth periods in six environments}

The results of the joint ANOVA analysis revealed that significant differences in the mean values of the HD or FD grouped by grades $0-5$ in all six environments (Table 2). Briefly, grade 5 exhibited the shortest length of the growth period, while grade 0 exhibited the greatest length. In detail, significant differences in the average values of growth period data were also found between different levels of each trait. The trend was similar to that revealed by the joint ANOVA results. Generally, the smaller the value of the $\mathrm{HD}$ or $\mathrm{FD}$ is, the greater the value of the grade (Fig. 2).

Significant negative correlations were detected between growth habit and growth period in six environments (Table 2, $p<0.01$ ). According to the results of joint variance analysis, the mean values of $H D$ and $F D$ were also correlated with growth habit; the Pearson correlation coefficients were -0.915 and -0.886 , respectively. Generally, these results also indicated that HD were more tightly, though negatively, related with growth habits. Furthermore, the range of correlation coefficients in six environments were from -0.813 (FD_15_ZMD) to -0.938 (HD_15_ZZ). Thus, we could conclude that the duration of the heading and flowering time of cultivars was tightly associated with growth habits (Additional file 1: Table S2).

\section{Distribution frequency of Vrn-1 alleles in varieties}

Because no polymorphisms were found in the $V r n-A 1$ and Vrn-B3 alleles, we focused on Vrn-B1 and Vrn-D1. The distribution frequency order of the dominant alleles was Vrn-D1a (23.70\%)>Vrn-D1b (8.10\%) > Vrn-B1a $(2.50 \%)>V r n-B 1 b(2.00 \%)$ (Table 3$)$. Only one accession was found to carry Vrn-B1b+Vrn-D1a, and 125 accessions presented no dominant alleles. We also used the "consistency index" to evaluate the reliability between the allelic detection and speculated results as described by Stelmakh [19]. According to Stelmakh's report, the accessions that contained at least one dominant allele were classified as spring types, whereas they were classified as winter types if they had three recessive alleles. Then, we found that nine accessions harbouring dominant $V r n-B 1 a$ or $V r n-B 1 b$ alleles as well as one accession harbouring $V r n-B 1 b+V r n-D 1 a$ exhibited the highest rate of consistency (100\%). Therefore, these accessions were classified as spring types, which were identical to the results of identification of growth habit in this research. The genotype rate of vrn-B1+vrn-D1 (63.1\%) dominated in all tested panels, and its consistency $(96.8 \%$ or $95.2 \%)$ was also higher than that of Vrn-D1a (48.9\%) and Vrn-D1b (25.0\%). Therefore, Vrn-D1, especially $V r n-D 1 b$, could not accurately estimate the growth habit.

\section{Effects of Vrn-1 combinations on HD and FD}

The effects of Vrn-B1+Vrn-D1 combinations concerning $\mathrm{HD}$ and FD were examined. In total, there were 6 different types of genotypes grouped by combinations. Among them, 125 accessions had double-recessive $v r n-B 1+v r n-D 1$ alleles. However, Vrn-B1a+vrn-D1, $V r n-B 1 b+v r n-D 1, v r n-B 1+V r n-D 1 a, v r n-B 1+V r n-D 1 b$ alleles were carried by $5,4,47$ and 16 varieties, respectively. Only 1 accession harboured double-dominant $V r n-B 1 b+V r n-D 1 a$ alleles. Least significant range (LSR, a method of multiple comparison) tests revealed significant differences among the six groups with respect to the mean values of $\mathrm{HD}$ and $\mathrm{FD}$ in almost all environments $(P$ $<0.05)$, with the exception that LSR tests for FD_15_ZMD were not significant (highlightedwith yellow). However, no significant differences in the mean values of each group across environments were revealed by the joint ANOVA results (highlighted with green) (Table 4).

Because the low frequency of the Vrn-B1b+Vrn-D1a type $(0.5 \%)$ made it difficult to exactly compare this allelic combination with other genotypes, we focused on the other five combinations. With respect to their effects, we found that accessions with the vrn-B1+ $v r n-D 1$ genotype presented the latest HD and FD (178.9 d), while varieties that harboured the Vrn-B1b+vrn-D1 
Table 1 The phenotypic variants in winter/spring growth habits and allelic variants on $\mathrm{Vrn}$-1 alleles

\begin{tabular}{|c|c|c|c|c|c|c|c|c|c|}
\hline ID & Taxa & Registered & Origination & W/S_2016 & W/S_2015 & Vrn-A1 & Vrn-B1 & Vrn-D1 & Vrn-B3 \\
\hline QZ01 & Shanyou 225 & winter & Shaanxi & 5 & 5 & $v r n-A 1$ & $v r n-B 1$ & Vrn-D1a & $v r n-B 3$ \\
\hline QZ02 & Aikang 58 & semi-winter & Henan & 3 & 3 & $v r n-A 1$ & $v r n-B 1$ & $v r n-D 1$ & $v r n-B 3$ \\
\hline QZ03 & Zhoumai 24 & semi-winter & Henan & 2 & 2 & $v r n-A 1$ & $v r n-B 1$ & $v r n-D 1$ & $v r n-B 3$ \\
\hline QZO4 & Zaoxiang 158 & semi-winter & Henan & 2 & 2 & $v r n-A 1$ & $v r n-B 1$ & $v r n-D 1$ & $v r n-B 3$ \\
\hline QZ05 & Zhengmai 366 & semi-winter & Henan & 2 & 2 & $v r n-A 1$ & $v r n-B 1$ & $v r n-D 1$ & $v r n-B 3$ \\
\hline QZ06 & Bainong 418 & semi-winter & Henan & 4 & 4 & $v r n-A 1$ & $v r n-B 1$ & $v r n-D 1$ & $v r n-B 3$ \\
\hline QZ07 & Taihemai 1 & semi-winter & Henan & 1 & 1 & $v r n-A 1$ & $v r n-B 1$ & $v r n-D 1$ & $v r n-B 3$ \\
\hline QZ08 & Anmai 8 & semi-winter & Henan & 1 & 1 & $v r n-A 1$ & $v r n-B 1$ & $v r n-D 1$ & $v r n-B 3$ \\
\hline QZ09 & Huaimai 05159 & semi-winter & Henan & 4 & 4 & $v r n-A 1$ & $v r n-B 1$ & vrn-D1 & $v r n-B 3$ \\
\hline QZ10 & Zhoumai 16 & semi-winter & Henan & 1 & 1 & $v r n-A 1$ & $v r n-B 1$ & vrn-D1 & $v r n-B 3$ \\
\hline QZ11 & Xinmai 18 & semi-winter & Henan & 1 & 1 & $v r n-A 1$ & $v r n-B 1$ & $v r n-D 1$ & $v r n-B 3$ \\
\hline QZ12 & Xianmai 13 & weak spring & Henan & 5 & 5 & $v r n-A 1$ & $v r n-B 1$ & Vrn-Dla & $v r n-B 3$ \\
\hline QZ13 & Yandian 9433 & semi-winter & Henan & 1 & 1 & $v r n-A 1$ & $v r n-B 1$ & $v r n-D 1$ & $v r n-B 3$ \\
\hline QZ14 & Zhongchuang 805 & weak spring & Henan & 1 & 1 & $v r n-A 1$ & $v r n-B 1$ & vrn-D1 & $v r n-B 3$ \\
\hline QZ15 & Zhengyumai 9987 & semi-winter & Henan & 3 & 3 & $v r n-A 1$ & $v r n-B 1$ & Vrn-D1a & $v r n-B 3$ \\
\hline QZ16 & Luomai 31 & semi-winter & Henan & 3 & 3 & $v r n-A 1$ & $v r n-B 1$ & $v r n-D 1$ & $v r n-B 3$ \\
\hline QZ17 & Yimai 6 & semi-winter & Henan & 2 & 2 & $v r n-A 1$ & $v r n-B 1$ & Vrn-D1b & $v r n-B 3$ \\
\hline QZ18 & Zhengzhong 17 & semi-winter & Henan & 3 & 3 & $v r n-A 1$ & $v r n-B 1$ & Vrn-D1a & $v r n-B 3$ \\
\hline QZ19 & Fanmai 803 & semi-winter & Henan & 1 & 1 & $v r n-A 1$ & $v r n-B 1$ & $v r n-D 1$ & $v r n-B 3$ \\
\hline QZ20 & Zhoumai 27 & semi-winter & Henan & 2 & 2 & $v r n-A 1$ & $v r n-B 1$ & $v r n-D 1$ & $v r n-B 3$ \\
\hline QZ21 & Luomai 28 & semi-winter & Henan & 4 & 4 & $v r n-A 1$ & $v r n-B 1$ & $v r n-D 1$ & $v r n-B 3$ \\
\hline QZ22 & Yunong 982 & semi-winter & Henan & 1 & 1 & $v r n-A 1$ & $v r n-B 1$ & $v r n-D 1$ & $v r n-B 3$ \\
\hline QZ23 & Zhengmai 1023 & semi-winter & Henan & 0 & 0 & $v r n-A 1$ & $v r n-B 1$ & vrn-D1 & $v r n-B 3$ \\
\hline QZ24 & BN 160 & semi-winter & Henan & 1 & 1 & $v r n-A 1$ & $v r n-B 1$ & vrn-D1 & $v r n-B 3$ \\
\hline QZ25 & 04 zhong 36 & weak spring & Henan & 5 & 5 & $v r n-A 1$ & $v r n-B 1$ & Vrn-D1a & $v r n-B 3$ \\
\hline QZ26 & Lankao198 & weak spring & Henan & 5 & 5 & $v r n-A 1$ & $v r n-B 1$ & Vrn-D1b & $v r n-B 3$ \\
\hline QZ27 & Fengdecunmai 5 & semi-winter & Henan & 3 & 3 & $v r n-A 1$ & $v r n-B 1$ & $v r n-D 1$ & $v r n-B 3$ \\
\hline QZ28 & Guoyu 101 & semi-winter & Henan & 1 & 1 & $v r n-A 1$ & $v r n-B 1$ & vrn-D1 & $v r n-B 3$ \\
\hline QZ29 & Wen 0418 & semi-winter & Henan & 1 & 1 & $v r n-A 1$ & $v r n-B 1$ & $v r n-D 1$ & $v r n-B 3$ \\
\hline QZ30 & Yunong 202 & semi-winter & Henan & 4 & 4 & $v r n-A 1$ & $v r n-B 1$ & $v r n-D 1$ & $v r n-B 3$ \\
\hline QZ31 & Zhongyu 9307 & semi-winter & Henan & 1 & 1 & $v r n-A 1$ & $v r n-B 1$ & $v r n-D 1$ & $v r n-B 3$ \\
\hline QZ32 & Ruzhou 0319 & semi-winter & Henan & 2 & 2 & $v r n-A 1$ & $v r n-B 1$ & $v r n-D 1$ & $v r n-B 3$ \\
\hline QZ33 & Yumai 41 & semi-winter & Henan & 0 & 0 & $v r n-A 1$ & $v r n-B 1$ & $v r n-D 1$ & $v r n-B 3$ \\
\hline QZ34 & Yumai 55 & semi-winter & Henan & 1 & 1 & $v r n-A 1$ & $v r n-B 1$ & $v r n-D 1$ & $v r n-B 3$ \\
\hline QZ35 & Yunong 186 & semi-winter & Henan & 4 & 4 & $v r n-A 1$ & $v r n-B 1$ & $v r n-D 1$ & $v r n-B 3$ \\
\hline QZ36 & Junda 106 & semi-winter & Henan & 1 & 1 & $v r n-A 1$ & $v r n-B 1$ & vrn-D1 & $v r n-B 3$ \\
\hline QZ37 & Yumai 49 & semi-winter & Henan & 1 & 1 & $v r n-A 1$ & $v r n-B 1$ & $v r n-D 1$ & $v r n-B 3$ \\
\hline QZ38 & Zhengmai 7698 & semi-winter & Henan & 4 & 4 & $v r n-A 1$ & $v r n-B 1$ & vrn-D1 & $v r n-B 3$ \\
\hline QZ39 & Lunxuan 1298 & semi-winter & Henan & 1 & 1 & $v r n-A 1$ & $v r n-B 1$ & $v r n-D 1$ & $v r n-B 3$ \\
\hline QZ40 & Mengmai 023 & weak spring & Henan & 5 & 5 & $v r n-A 1$ & Vrn-B1a & $v r n-D 1$ & $v r n-B 3$ \\
\hline QZ41 & Luomai 23 & semi-winter & Henan & 5 & 5 & $v r n-A 1$ & $v r n-B 1$ & Vrn-D1a & $v r n-B 3$ \\
\hline QZ42 & Jiyanmai 7 & semi-winter & Henan & 2 & 2 & $v r n-A 1$ & $v r n-B 1$ & $v r n-D 1$ & $v r n-B 3$ \\
\hline QZ43 & Fengdecunmai 8 & semi-winter & Henan & 1 & 1 & $v r n-A 1$ & $v r n-B 1$ & $v r n-D 1$ & $v r n-B 3$ \\
\hline QZ44 & Zhoumai 9 & semi-winter & Henan & 1 & 1 & $v r n-A 1$ & $v r n-B 1$ & vrn-D1 & $v r n-B 3$ \\
\hline
\end{tabular}


Table 1 The phenotypic variants in winter/spring growth habits and allelic variants on Vrn-1 alleles (Continued)

\begin{tabular}{|c|c|c|c|c|c|c|c|c|c|}
\hline ID & Taxa & Registered & Origination & W/S_2016 & W/S_2015 & Vrn-A1 & Vrn-B1 & Vrn-D1 & $V r n-B 3$ \\
\hline QZ45 & Zhengyumai 0519 & semi-winter & Henan & 1 & 1 & $v r n-A 1$ & $v r n-B 1$ & $v r n-D 1$ & $v r n-B 3$ \\
\hline QZ46 & Xuke 316 & semi-winter & Henan & 4 & 4 & $v r n-A 1$ & $v r n-B 1$ & Vrn-D1a & $v r n-B 3$ \\
\hline QZ47 & Luomai 18 & weak spring & Henan & 5 & 5 & $v r n-A 1$ & $v r n-B 1$ & Vrn-D1a & $v r n-B 3$ \\
\hline QZ48 & Xinong 979 & semi-winter & Henan & 5 & 4 & $v r n-A 1$ & $v r n-B 1$ & $v r n-D 1$ & $v r n-B 3$ \\
\hline QZ49 & Cunmai 11 & semi-winter & Henan & 2 & 2 & $v r n-A 1$ & $v r n-B 1$ & vrn-D1 & $v r n-B 3$ \\
\hline QZ50 & Fengdecunmai 12 & semi-winter & Henan & 2 & 2 & $v r n-A 1$ & $v r n-B 1$ & $v r n-D 1$ & $v r n-B 3$ \\
\hline QZ51 & Huaichuan 919 & semi-winter & Henan & 2 & 2 & $v r n-A 1$ & $v r n-B 1$ & Vrn-D1a & $v r n-B 3$ \\
\hline QZ52 & Zou 8425B & semi-winter & Henan & 4 & 4 & $v r n-A 1$ & $v r n-B 1$ & Vrn-D1a & $v r n-B 3$ \\
\hline QZ53 & Yunong 211 & semi-winter & Henan & 1 & 1 & $v r n-A 1$ & $v r n-B 1$ & $v r n-D 1$ & $v r n-B 3$ \\
\hline QZ54 & LS 6109 & semi-winter & Shandong & 1 & 1 & $v r n-A 1$ & $v r n-B 1$ & vrn-D1 & $v r n-B 3$ \\
\hline QZ55 & Lankao 182 & semi-winter & Henan & 1 & 1 & $v r n-A 1$ & $v r n-B 1$ & $v r n-D 1$ & $v r n-B 3$ \\
\hline QZ56 & Xumai 1242 & semi-winter & Henan & 2 & 2 & $v r n-A 1$ & $v r n-B 1$ & $v r n-D 1$ & $v r n-B 3$ \\
\hline QZ57 & Zhoumai 26 & semi-winter & Henan & 5 & 5 & $v r n-A 1$ & $v r n-B 1$ & $v r n-D 1$ & $v r n-B 3$ \\
\hline QZ58 & Yamai 1 & weak spring & Henan & 5 & 5 & $v r n-A 1$ & Vrn-B1a & vrn-D1 & $v r n-B 3$ \\
\hline QZ59 & Yujiao 5 & semi-winter & Henan & 0 & 0 & $v r n-A 1$ & $v r n-B 1$ & $v r n-D 1$ & $v r n-B 3$ \\
\hline QZ60 & Fanmai 11 & semi-winter & Henan & 4 & 4 & $v r n-A 1$ & $v r n-B 1$ & $v r n-D 1$ & $v r n-B 3$ \\
\hline QZ61 & Zhengmai 103 & semi-winter & Henan & 4 & 4 & $v r n-A 1$ & $v r n-B 1$ & Vrn-D1a & $v r n-B 3$ \\
\hline QZ62 & Pumai 053 & semi-winter & Henan & 1 & 1 & $v r n-A 1$ & $v r n-B 1$ & $v r n-D 1$ & $v r n-B 3$ \\
\hline QZ63 & Tunfeng 802 & semi-winter & Henan & 3 & 3 & $v r n-A 1$ & $v r n-B 1$ & vrn-D1 & $v r n-B 3$ \\
\hline QZ64 & Fengdecunmai 1 & semi-winter & Henan & 4 & 4 & $v r n-A 1$ & $v r n-B 1$ & vrn-D1 & $v r n-B 3$ \\
\hline QZ65 & Pu2056 & semi-winter & Henan & 5 & 5 & $v r n-A 1$ & $V r n-B 1 b$ & $v r n-D 1$ & $v r n-B 3$ \\
\hline QZ66 & Xinmai 19 & semi-winter & Henan & 4 & 4 & $v r n-A 1$ & $v r n-B 1$ & $v r n-D 1$ & $v r n-B 3$ \\
\hline QZ67 & Wenliang 1 & semi-winter & Henan & 1 & 1 & $v r n-A 1$ & $v r n-B 1$ & $v r n-D 1$ & $v r n-B 3$ \\
\hline QZ68 & Zhongyu 9302 & semi-winter & Henan & 1 & 1 & vrn-A1 & $v r n-B 1$ & vrn-D1 & $v r n-B 3$ \\
\hline QZ69 & Zhengmai 583 & semi-winter & Henan & 1 & 1 & $v r n-A 1$ & $v r n-B 1$ & $v r n-D 1$ & $v r n-B 3$ \\
\hline QZ70 & Hengguan 35 & semi-winter & Henan & 5 & 5 & $v r n-A 1$ & $v r n-B 1$ & Vrn-D1b & $v r n-B 3$ \\
\hline QZ71 & Luomai 24 & weak spring & Henan & 5 & 5 & $v r n-A 1$ & $v r n-B 1$ & Vrn-D1a & $v r n-B 3$ \\
\hline QZ72 & Yunong 416 & semi-winter & Henan & 5 & 5 & $v r n-A 1$ & $v r n-B 1$ & $v r n-D 1$ & $v r n-B 3$ \\
\hline QZ73 & Zhoumai 22 & semi-winter & Henan & 1 & 1 & $v r n-A 1$ & $v r n-B 1$ & $v r n-D 1$ & $v r n-B 3$ \\
\hline QZ74 & Yumai 52 & semi-winter & Henan & 1 & 1 & $v r n-A 1$ & $v r n-B 1$ & $v r n-D 1$ & $v r n-B 3$ \\
\hline QZ75 & FS 059 & semi-winter & Henan & 1 & 1 & $v r n-A 1$ & $v r n-B 1$ & $v r n-D 1$ & $v r n-B 3$ \\
\hline QZ76 & Pingan 11 & semi-winter & Henan & 1 & 1 & $v r n-A 1$ & $v r n-B 1$ & $v r n-D 1$ & $v r n-B 3$ \\
\hline QZ77 & Bonong 6 & weak spring & Henan & 5 & 5 & $v r n-A 1$ & $v r n-B 1$ & vrn-D1 & $v r n-B 3$ \\
\hline QZ78 & Zhoumai 18 & semi-winter & Henan & 1 & 1 & vrn-Al & $v r n-B 1$ & $v r n-D 1$ & $v r n-B 3$ \\
\hline QZ79 & Xuke 168 & semi-winter & Henan & 3 & 3 & $v r n-A 1$ & $v r n-B 1$ & Vrn-D1a & $v r n-B 3$ \\
\hline QZ80 & Pumai 10 & semi-winter & Henan & 3 & 3 & $v r n-A 1$ & $v r n-B 1$ & Vrn-D1a & $v r n-B 3$ \\
\hline QZ81 & Xumai 0054 & semi-winter & Jiangsu & 1 & 1 & $v r n-A 1$ & $v r n-B 1$ & vrn-D1 & $v r n-B 3$ \\
\hline QZ82 & Zhengyou 6 & semi-winter & Henan & 5 & 5 & vrn-A1 & Vrn-B1a & vrn-D1 & $v r n-B 3$ \\
\hline QZ83 & Yunong 9901 & weak spring & Henan & 5 & 5 & $v r n-A 1$ & $v r n-B 1$ & Vrn-D1a & $v r n-B 3$ \\
\hline QZ84 & Zhengmai 0856 & semi-winter & Henan & 4 & 4 & $v r n-A 1$ & $v r n-B 1$ & $v r n-D 1$ & $v r n-B 3$ \\
\hline QZ85 & Luomai 8 & semi-winter & Henan & 1 & 1 & $v r n-A 1$ & $v r n-B 1$ & $v r n-D 1$ & $v r n-B 3$ \\
\hline QZ86 & Zhoumai 13 & semi-winter & Henan & 2 & 2 & $v r n-A 1$ & $v r n-B 1$ & Vrn-D1a & $v r n-B 3$ \\
\hline QZ87 & Luo 10 T07 & semi-winter & Henan & 2 & 2 & $v r n-A 1$ & $v r n-B 1$ & $v r n-D 1$ & $v r n-B 3$ \\
\hline QZ88 & Yuanyu 3 & weak spring & Henan & 5 & 5 & $v r n-A 1$ & $v r n-B 1$ & Vrn-D1a & $v r n-B 3$ \\
\hline
\end{tabular}


Table 1 The phenotypic variants in winter/spring growth habits and allelic variants on Vrn-1 alleles (Continued)

\begin{tabular}{|c|c|c|c|c|c|c|c|c|c|}
\hline ID & Taxa & Registered & Origination & W/S_2016 & W/S_2015 & Vrn-A1 & $V r n-B 1$ & Vrn-D1 & $V r n-B 3$ \\
\hline QZ89 & Fengdecunmai 10 & semi-winter & Henan & 4 & 4 & $v r n-A 1$ & $v r n-B 1$ & $v r n-D 1$ & $v r n-B 3$ \\
\hline QZ90 & Hongmai 118 & semi-winter & Henan & 1 & 1 & $v r n-A 1$ & $v r n-B 1$ & vrn-D1 & $v r n-B 3$ \\
\hline QZ91 & Yumai 14 You & semi-winter & Henan & 4 & 4 & $v r n-A 1$ & $v r n-B 1$ & Vrn-D1a & $v r n-B 3$ \\
\hline QZ92 & Zhoumai 19 & semi-winter & Henan & 4 & 4 & $v r n-A 1$ & $v r n-B 1$ & Vrn-D1b & $v r n-B 3$ \\
\hline QZ93 & Guomai 301 & semi-winter & Henan & 0 & 0 & $v r n-A 1$ & $v r n-B 1$ & vrn-D1 & $v r n-B 3$ \\
\hline QZ94 & Zhengmai 113 & weak spring & Henan & 5 & 5 & $v r n-A 1$ & $v r n-B 1$ & Vrn-Dla & $v r n-B 3$ \\
\hline QZ95 & Pumai 9 & weak spring & Henan & 5 & 5 & $v r n-A 1$ & $v r n-B 1$ & Vrn-D1a & $v r n-B 3$ \\
\hline QZ96 & Pingan 8 & semi-winter & Henan & 1 & 1 & $v r n-A 1$ & $v r n-B 1$ & $v r n-D 1$ & $v r n-B 3$ \\
\hline QZ97 & Xinmai 20 & weak spring & Henan & 5 & 5 & $v r n-A 1$ & $v r n-B 1$ & Vrn-Dla & $v r n-B 3$ \\
\hline QZ98 & Zhongmai 875 & semi-winter & Beijing & 2 & 2 & $v r n-A 1$ & $v r n-B 1$ & $v r n-D 1$ & $v r n-B 3$ \\
\hline QZ99 & Zhongyanmai 0708 & weak spring & Jiangsu & 3 & 3 & $v r n-A 1$ & $v r n-B 1$ & $v r n-D 1$ & $v r n-B 3$ \\
\hline QZ100 & Zhongmai 1 & semi-winter & Henan & 1 & 1 & $v r n-A 1$ & $v r n-B 1$ & $v r n-D 1$ & $v r n-B 3$ \\
\hline QZ101 & Nongda 1108 & semi-winter & Beijing & 1 & 1 & $v r n-A 1$ & $v r n-B 1$ & Vrn-D1a & $v r n-B 3$ \\
\hline QZ102 & Yumai 47 & weak spring & Henan & 5 & 2 & $v r n-A 1$ & $v r n-B 1$ & vrn-D1 & $v r n-B 3$ \\
\hline QZ103 & Zhengmai 98 & semi-winter & Henan & 2 & 2 & $v r n-A 1$ & $v r n-B 1$ & Vrn-D1a & $v r n-B 3$ \\
\hline QZ104 & Xinong 889 & semi-winter & Shaanxi & 2 & 2 & $v r n-A 1$ & $v r n-B 1$ & $v r n-D 1$ & $v r n-B 3$ \\
\hline QZ105 & Neixiang 188 & semi-winter & Henan & 2 & 2 & $v r n-A 1$ & $v r n-B 1$ & $v r n-D 1$ & $v r n-B 3$ \\
\hline QZ106 & Jimai 22 & semi-winter & Shandong & 3 & 3 & $v r n-A 1$ & $v r n-B 1$ & $v r n-D 1$ & $v r n-B 3$ \\
\hline QZ107 & Fanmai 5 & semi-winter & Henan & 2 & 2 & $v r n-A 1$ & $v r n-B 1$ & Vrn-D1a & $v r n-B 3$ \\
\hline QZ108 & Huayu 198 & semi-winter & Henan & 0 & 0 & $v r n-A 1$ & $v r n-B 1$ & vrn-D1 & $v r n-B 3$ \\
\hline QZ109 & Huangming 116 & weak spring & Henan & 3 & 3 & $v r n-A 1$ & $v r n-B 1$ & $v r n-D 1$ & $v r n-B 3$ \\
\hline QZ110 & Zhengmai 9694 & semi-winter & Henan & 1 & 1 & $v r n-A 1$ & $v r n-B 1$ & $v r n-D 1$ & $v r n-B 3$ \\
\hline QZ111 & Yunong 949 & weak spring & Henan & 5 & 5 & $v r n-A 1$ & $v r n-B 1$ & Vrn-D1a & $v r n-B 3$ \\
\hline QZ112 & Shan 160 & semi-winter & Shaanxi & 3 & 3 & vrn-A1 & $v r n-B 1$ & vrn-D1 & $v r n-B 3$ \\
\hline QZ113 & Luomai 4 & semi-winter & Henan & 1 & 1 & $v r n-A 1$ & $v r n-B 1$ & $v r n-D 1$ & $v r n-B 3$ \\
\hline QZ114 & Luoxin 998 & semi-winter & Henan & 1 & 1 & $v r n-A 1$ & $v r n-B 1$ & Vrn-D1a & $v r n-B 3$ \\
\hline QZ115 & Yunong 201 & semi-winter & Henan & 1 & 1 & $v r n-A 1$ & $v r n-B 1$ & $v r n-D 1$ & $v r n-B 3$ \\
\hline QZ116 & Kaimai 21 & semi-winter & Henan & 1 & 1 & $v r n-A 1$ & $v r n-B 1$ & $v r n-D 1$ & $v r n-B 3$ \\
\hline QZ117 & Yubao 1 & semi-winter & Henan & 1 & 1 & $v r n-A 1$ & $v r n-B 1$ & Vrn-D1b & $v r n-B 3$ \\
\hline QZ118 & Bainong 207 & semi-winter & Henan & 2 & 2 & $v r n-A 1$ & $v r n-B 1$ & $v r n-D 1$ & $v r n-B 3$ \\
\hline QZ119 & Luomai 21 & semi-winter & Henan & 3 & 3 & $v r n-A 1$ & $v r n-B 1$ & Vrn-D1b & $v r n-B 3$ \\
\hline QZ120 & Zhengmai 101 & weak spring & Henan & 1 & 1 & $v r n-A 1$ & $v r n-B 1$ & $v r n-D 1$ & $v r n-B 3$ \\
\hline QZ121 & Xinmai 9 & semi-winter & Henan & 1 & 1 & $v r n-A 1$ & $v r n-B 1$ & vrn-D1 & $v r n-B 3$ \\
\hline QZ122 & Xinmai 208 & weak spring & Henan & 5 & 5 & vrn-Al & $v r n-B 1$ & Vrn-D1b & $v r n-B 3$ \\
\hline QZ123 & Yumai 34 & weak spring & Henan & 5 & 5 & $v r n-A 1$ & Vrn-B1b & $v r n-D 1$ & $v r n-B 3$ \\
\hline QZ124 & Kaimai 18 & semi-winter & Henan & 2 & 2 & $v r n-A 1$ & $v r n-B 1$ & $v r n-D 1$ & $v r n-B 3$ \\
\hline QZ125 & Xuke 793 & semi-winter & Henan & 1 & 1 & $v r n-A 1$ & $v r n-B 1$ & vrn-D1 & $v r n-B 3$ \\
\hline QZ126 & Fanmai 7030 & semi-winter & Henan & 4 & 4 & vrn-A1 & $v r n-B 1$ & vrn-D1 & $v r n-B 3$ \\
\hline QZ127 & Shi 4185 & semi-winter & Hebei & 3 & 3 & $v r n-A 1$ & $v r n-B 1$ & Vrn-D1a & $v r n-B 3$ \\
\hline QZ128 & Xin 0208 & semi-winter & Henan & 2 & 2 & $v r n-A 1$ & $v r n-B 1$ & $v r n-D 1$ & $v r n-B 3$ \\
\hline QZ129 & Zhengmai 379 & semi-winter & Henan & 3 & 3 & $v r n-A 1$ & $v r n-B 1$ & Vrn-D1a & $v r n-B 3$ \\
\hline QZ130 & Jimai 20 & semi-winter & Shandong & 4 & 4 & $v r n-A 1$ & $v r n-B 1$ & $v r n-D 1$ & $v r n-B 3$ \\
\hline QZ131 & Qiule 2122 & semi-winter & Henan & 4 & 4 & $v r n-A 1$ & $v r n-B 1$ & Vrn-D1a & $v r n-B 3$ \\
\hline QZ132 & Zhoumai 23 & weak spring & Henan & 4 & 4 & vrn-A1 & $v r n-B 1$ & Vrn-D1a & $v r n-B 3$ \\
\hline
\end{tabular}


Table 1 The phenotypic variants in winter/spring growth habits and allelic variants on Vrn-1 alleles (Continued)

\begin{tabular}{|c|c|c|c|c|c|c|c|c|c|}
\hline ID & Taxa & Registered & Origination & W/S_2016 & W/S_2015 & Vrn-A1 & $V r n-B 1$ & Vrn-D1 & $V_{r n}-B 3$ \\
\hline QZ133 & Zhengyumai 043 & semi-winter & Henan & 1 & 1 & $v r n-A 1$ & $v r n-B 1$ & Vrn-D1a & $v r n-B 3$ \\
\hline QZ134 & Zhengmai 004 & semi-winter & Henan & 3 & 3 & $v r n-A 1$ & $v r n-B 1$ & Vrn-D1a & $v r n-B 3$ \\
\hline QZ135 & Wennong 14 & semi-winter & Shandong & 2 & 2 & $v r n-A 1$ & $v r n-B 1$ & $v r n-D 1$ & $v r n-B 3$ \\
\hline QZ136 & Xinhan 1 & semi-winter & Henan & 4 & 4 & $v r n-A 1$ & $v r n-B 1$ & Vrn-D1a & $v r n-B 3$ \\
\hline QZ137 & Yumai 18 & weak spring & Henan & 5 & 5 & $v r n-A 1$ & $v r n-B 1$ & Vrn-D1a & $v r n-B 3$ \\
\hline QZ138 & Xuke 415 & semi-winter & Henan & 1 & 1 & $v r n-A 1$ & $v r n-B 1$ & $v r n-D 1$ & $v r n-B 3$ \\
\hline QZ139 & Pingan 9 & semi-winter & Henan & 1 & 1 & $v r n-A 1$ & $v r n-B 1$ & $v r n-D 1$ & $v r n-B 3$ \\
\hline QZ140 & Shangmai 156 & semi-winter & Henan & 1 & 1 & $v r n-A 1$ & $v r n-B 1$ & Vrn-D1a & $v r n-B 3$ \\
\hline QZ141 & Xinong 9871 & semi-winter & Shaanxi & 5 & 5 & $v r n-A 1$ & $v r n-B 1$ & Vrn-Dla & $v r n-B 3$ \\
\hline QZ142 & Pingan 3 & semi-winter & Henan & 1 & 1 & $v r n-A 1$ & $v r n-B 1$ & vrn-D1 & $v r n-B 3$ \\
\hline QZ143 & Zhongyu 12 & semi-winter & Henan & 2 & 2 & $v r n-A 1$ & $v r n-B 1$ & Vrn-D1b & $v r n-B 3$ \\
\hline QZ144 & Yumai 58 & semi-winter & Henan & 1 & 1 & $v r n-A 1$ & $v r n-B 1$ & $v r n-D 1$ & $v r n-B 3$ \\
\hline QZ145 & Xun 9917 & semi-winter & Henan & 4 & 4 & $v r n-A 1$ & $v r n-B 1$ & Vrn-D1b & $v r n-B 3$ \\
\hline QZ146 & Ping'an 6 & weak spring & Henan & 5 & 5 & $v r n-A 1$ & Vrn-B1b & Vrn-D1a & $v r n-B 3$ \\
\hline QZ147 & Xinmai 26 & semi-winter & Henan & 1 & 1 & $v r n-A 1$ & $v r n-B 1$ & vrn-D1 & $v r n-B 3$ \\
\hline QZ148 & Lankaoaizao 8 & weak spring & Henan & 2 & 2 & $v r n-A 1$ & $v r n-B 1$ & vrn-D1 & $v r n-B 3$ \\
\hline QZ149 & Fengyou 6 & weak spring & Henan & 5 & 5 & $v r n-A 1$ & Vrn-B1a & $v r n-D 1$ & $v r n-B 3$ \\
\hline QZ150 & Huaimai 0882 & semi-winter & Jiangsu & 1 & 1 & $v r n-A 1$ & $v r n-B 1$ & $v r n-D 1$ & $v r n-B 3$ \\
\hline QZ151 & Taikong 6 & weak spring & Henan & 1 & 1 & $v r n-A 1$ & $v r n-B 1$ & vrn-D1 & $v r n-B 3$ \\
\hline QZ152 & Xinong 529 & weak spring & Shaanxi & 5 & 5 & $v r n-A 1$ & $v r n-B 1$ & Vrn-D1a & $v r n-B 3$ \\
\hline QZ153 & Yumai 51 & weak spring & Henan & 5 & 5 & $v r n-A l$ & $v r n-B 1$ & Vrn-Dla & $v r n-B 3$ \\
\hline QZ154 & Luo 6073 & semi-winter & Henan & 1 & 1 & $v r n-A 1$ & $v r n-B 1$ & vrn-D1 & $v r n-B 3$ \\
\hline QZ155 & Bainong 64 & semi-winter & Henan & 1 & 1 & $v r n-A 1$ & $v r n-B 1$ & $v r n-D 1$ & $v r n-B 3$ \\
\hline QZ156 & Jinan 17 & winter & Shandong & 2 & 2 & vrn-A1 & $v r n-B 1$ & $v r n-D 1$ & $v r n-B 3$ \\
\hline QZ157 & Yanzhan 4110 & weak spring & Henan & 5 & 5 & $v r n-A 1$ & $v r n-B 1$ & Vrn-D1a & $v r n-B 3$ \\
\hline QZ158 & Weilai 0818 & semi-winter & Anhui & 1 & 1 & $v r n-A 1$ & $v r n-B 1$ & vrn-D1 & $v r n-B 3$ \\
\hline QZ159 & Yanke 028 & weak spring & Henan & 5 & 5 & $v r n-A 1$ & Vrn-B1a & $v r n-D 1$ & $v r n-B 3$ \\
\hline QZ160 & $86(79)-128$ & semi-winter & Henan & 4 & 4 & $v r n-A 1$ & $v r n-B 1$ & vrn-D1 & $v r n-B 3$ \\
\hline QZ161 & Yanmai864 & weak spring & Henan & 5 & 5 & vrn-A1 & Vrn-B1b & vrn-D1 & $v r n-B 3$ \\
\hline QZ162 & Xiaoyan 81 & semi-winter & Beijing & 2 & 2 & $v r n-A 1$ & $v r n-B 1$ & vrn-D1 & $v r n-B 3$ \\
\hline QZ163 & Liangxing 99 & semi-winter & Shandong & 2 & 2 & $v r n-A 1$ & $v r n-B 1$ & $v r n-D 1$ & $v r n-B 3$ \\
\hline QZ164 & Tainong 8968 & semi-winter & Shandong & 0 & 0 & $v r n-A 1$ & $v r n-B 1$ & $v r n-D 1$ & $v r n-B 3$ \\
\hline QZ165 & Huayumai 118 & semi-winter & Henan & 2 & 2 & $v r n-A 1$ & $v r n-B 1$ & vrn-D1 & $v r n-B 3$ \\
\hline QZ166 & Yanshi 16 & weak spring & Henan & 5 & 5 & $v r n-A 1$ & $v r n-B 1$ & Vrn-D1a & $v r n-B 3$ \\
\hline QZ167 & Xuke 1 & semi-winter & Henan & 1 & 1 & $v r n-A 1$ & $v r n-B 1$ & Vrn-D1a & $v r n-B 3$ \\
\hline QZ168 & Zhoumai 30 & semi-winter & Henan & 1 & 1 & $v r n-A 1$ & $v r n-B 1$ & Vrn-D1b & $v r n-B 3$ \\
\hline QZ169 & Yunong 4023 & semi-winter & Henan & 1 & 1 & vrn-A1 & $v r n-B 1$ & vrn-D1 & $v r n-B 3$ \\
\hline QZ170 & Zhengpinmai 8 & semi-winter & Henan & 1 & 1 & $v r n-A 1$ & $v r n-B 1$ & vrn-D1 & $v r n-B 3$ \\
\hline QZ171 & Zhengmai 9023 & weak spring & Henan & 5 & 5 & $v r n-A 1$ & Vrn-B1b & vrn-D1 & $v r n-B 3$ \\
\hline QZ172 & Shiluan 02-1 & semi-winter & Hebei & 0 & 0 & $v r n-A 1$ & $v r n-B 1$ & $v r n-D 1$ & $v r n-B 3$ \\
\hline QZ173 & Aifeng 3 & winter & Shaanxi & 1 & 1 & vrn-A1 & $v r n-B 1$ & Vrn-D1b & $v r n-B 3$ \\
\hline QZ174 & Han 6172 & winter & Hebei & 2 & 2 & $v r n-A 1$ & $v r n-B 1$ & vrn-D1 & $v r n-B 3$ \\
\hline QZ175 & Luohan 3 & semi-winter & Henan & 4 & 4 & $v r n-A 1$ & $v r n-B 1$ & $v r n-D 1$ & $v r n-B 3$ \\
\hline QZ176 & Yannong 19 & winter & Shandong & 0 & 0 & $v r n-A 1$ & $v r n-B 1$ & $v r n-D 1$ & $v r n-B 3$ \\
\hline
\end{tabular}


Table 1 The phenotypic variants in winter/spring growth habits and allelic variants on Vrn-1 alleles (Continued)

\begin{tabular}{|c|c|c|c|c|c|c|c|c|c|}
\hline ID & Taxa & Registered & Origination & W/S_2016 & W/S_2015 & Vrn-Al & Vrn-B1 & Vrn-D1 & Vrn-B3 \\
\hline QZ177 & Huaimai 19 & weak spring & Jiangsu & 1 & 1 & $v r n-A 1$ & $v r n-B 1$ & vrn-D1 & $v r n-B 3$ \\
\hline QZ178 & Yanmai 8911 & semi-winter & Shaanxi & 1 & 1 & $v r n-A 1$ & $v r n-B 1$ & Vrn-D1b & $v r n-B 3$ \\
\hline QZ179 & Bainong 3217 & weak winter & Henan & 5 & 5 & $v r n-A 1$ & $v r n-B 1$ & Vrn-D1a & $v r n-B 3$ \\
\hline QZ180 & Yumai 68 & semi-winter & Henan & 1 & 1 & $v r n-A 1$ & $v r n-B 1$ & $v r n-D 1$ & $v r n-B 3$ \\
\hline QZ181 & Yumai 2 & semi-winter & Henan & 1 & 1 & $v r n-A 1$ & $v r n-B 1$ & vrn-D1 & $v r n-B 3$ \\
\hline QZ182 & Gaocheng 8901 & semi-winter & Hebei & 1 & 1 & $v r n-A 1$ & $v r n-B 1$ & Vrn-D1a & $v r n-B 3$ \\
\hline QZ183 & Huarui 00712 & semi-winter & Jiangsu & 5 & 5 & $v r n-A 1$ & $v r n-B 1$ & Vrn-D1a & $v r n-B 3$ \\
\hline QZ184 & Shannong 7859 & semi-winter & Shaanxi & 4 & 4 & $v r n-A 1$ & $v r n-B 1$ & $v r n-D 1$ & $v r n-B 3$ \\
\hline QZ185 & Xiaoyan 22 & weak spring & Shaanxi & 1 & 1 & $v r n-A 1$ & $v r n-B 1$ & Vrn-D1b & $v r n-B 3$ \\
\hline QZ186 & Ruiquanmai 168 & semi-winter & Henan & 4 & 4 & $v r n-A 1$ & $v r n-B 1$ & Vrn-D1b & $v r n-B 3$ \\
\hline QZ187 & Hemai 26 & semi-winter & Henan & 0 & 0 & $v r n-A 1$ & $v r n-B 1$ & $v r n-D 1$ & $v r n-B 3$ \\
\hline QZ188 & Yan 99,102 & semi-winter & Shandong & 2 & 2 & $v r n-A 1$ & $v r n-B 1$ & Vrn-D1b & $v r n-B 3$ \\
\hline QZ189 & Jihan 2 & semi-winter & Shandong & 5 & 5 & $v r n-A 1$ & $v r n-B 1$ & Vrn-D1b & $v r n-B 3$ \\
\hline QZ190 & Yumai 4 & weak spring & Henan & 4 & 4 & $v r n-A 1$ & $v r n-B 1$ & vrn-D1 & $v r n-B 3$ \\
\hline QZ191 & Luohan 6 & semi-winter & Henan & 5 & 5 & $v r n-A 1$ & $v r n-B 1$ & $v r n-D 1$ & $v r n-B 3$ \\
\hline QZ192 & Zhenghan 1 & semi-winter & Henan & 1 & 1 & $v r n-A 1$ & $v r n-B 1$ & $v r n-D 1$ & $v r n-B 3$ \\
\hline QZ193 & Jinmai 47 & semi-winter & Shanxi & 1 & 1 & $v r n-A 1$ & $v r n-B 1$ & $v r n-D 1$ & $v r n-B 3$ \\
\hline QZ194 & Yumai 8 & semi-winter & Henan & 1 & 1 & $v r n-A 1$ & $v r n-B 1$ & $v r n-D 1$ & $v r n-B 3$ \\
\hline QZ195 & Beijing 841 & winter & Beijing & 0 & 0 & $v r n-A 1$ & $v r n-B 1$ & vrn-D1 & $v r n-B 3$ \\
\hline QZ196 & Sumai 3 & weak spring & Jiangsu & 5 & 5 & $v r n-A 1$ & $v r n-B 1$ & Vrn-D1a & $v r n-B 3$ \\
\hline QZ197 & Nanda 2419 & weak spring & Jiangsu & 5 & 5 & $v r n-A 1$ & $v r n-B 1$ & Vrn-Dla & $v r n-B 3$ \\
\hline QZ198 & Chinese Spring & spring & Sichuan & 5 & 5 & $v r n-A 1$ & $v r n-B 1$ & Vrn-D1a & $v r n-B 3$ \\
\hline
\end{tabular}

W/S means winter/spring growth habits in 2015 or 2016

allelic combination presented the earliest $\mathrm{HD}$ and FD $(174.3 \mathrm{~d})$ as well as the shortest growth habit. This method could be useful for precisely identifying the differences in growth habits in each group individually. Then, analyses of $V r n-1$ combinations revealed that the effects of the dominant $V r n-B 1$ genotype on HD and FD were stronger than those of the dominant Vrn-D1 genotype. Finally, we concluded that the rank order of the

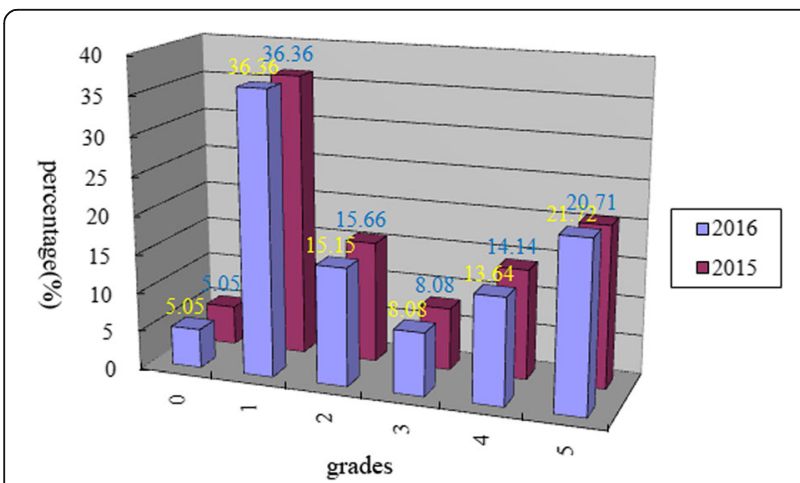

Fig. 1 Distribution rate in each grade of winter/spring growth habits among 198 wheat materials in 2015 and 2016 effects on the growth period was as follows: $V r n-B 1 b>$ $V r n-B 1 a>V r n-D 1 b>V r n-D 1 a>v r n-D 1=v r n-B 1$ (Table 4).

\section{Allelic variations of $P p d-1$ alleles}

No polymorphisms were found in the promoter of $P p d-A 1$ or $P p d-B 1$; thus, we focused on their internal variants. Here, variations in the junction sequences of $P p d-B 1$ were investigated to analyse their allelic variations and effects, which were considered copy number variations (CNVs) [28]. For Ppd-B1, the Ppd-B1a gene has three types in terms of CNV, accounting for $33.8 \%$ (Truncated CS type), 8.6\% (Intact CS type) and 35.3\% (Sonora 64 type) (Table 5). According to a previous report, the first two genotypes were named $P p d-B 1 c$ and the Sonora 64 type was named $P p d-B 1 a$ [30]. For variations within $P p d-B 1,6$ types of genotypic combinations were all detected because of their different types of combination. As to percentage, the "S: N: N" type constituted the largest proportion (34.34\%, "Sonora 64 type" only), while the percentages of "N: I: N" (1.01\%, "Intact CS type" only) and "S: N: T" (1.01\%, "Sonora 64 type + Truncated CS type" for short) were the lowest (Additional file 1: Table S2; Table 5). 
Table 2 The joint ANOVA analysis in HD and FD grouped by growth habit in six environments

\begin{tabular}{llllllll}
\hline Traits & 0 & 1 & 2 & 3 & 4 & 5 & $r$ \\
\hline HD_14_SQ & $183.3 \pm 2.0(\mathrm{a})$ & $182.3 \pm 2.2(\mathrm{ab})$ & $181.9 \pm 2.2(\mathrm{ab})$ & $181.3 \pm 1.9(\mathrm{~b})$ & $181.7 \pm 2.9(\mathrm{ab})$ & $178.5 \pm 2.9(\mathrm{c})$ & $-0.871^{*}$ \\
HD_15_SQ & $189.3 \pm 2.4(\mathrm{a})$ & $187.9 \pm 2.0(\mathrm{~b})$ & $186.9 \pm 2.0(\mathrm{~b})$ & $187.1 \pm 2.0(\mathrm{~b})$ & $186.5 \pm 2.1(\mathrm{~b})$ & $183.7 \pm 2.2(\mathrm{c})$ & $-0.922^{* *}$ \\
HD_14_ZMD & $172.0 \pm 1.8(\mathrm{a})$ & $171.3 \pm 1.4(\mathrm{a})$ & $171.3 \pm 1.2(\mathrm{a})$ & $171.3 \pm 1.7(\mathrm{a})$ & $171.0 \pm 1.7(\mathrm{a})$ & $169.9 \pm 1.1(\mathrm{~b})$ & $-0.884^{* *}$ \\
HD_15_ZMD & $159.1 \pm 1.5(\mathrm{a})$ & $158.3 \pm 1.1(\mathrm{~b})$ & $157.9 \pm 1.5(\mathrm{bc})$ & $157.9 \pm 1.1(\mathrm{bc})$ & $158.1 \pm 1.2(\mathrm{~b})$ & $157.2 \pm 1.5(\mathrm{c})$ & $-0.869^{*}$ \\
HD_14_ZZ & $181.7 \pm 1.9(\mathrm{a})$ & $180.9 \pm 1.1(\mathrm{ab})$ & $180.8 \pm 1.3(\mathrm{ab})$ & $180.1 \pm 1.3(\mathrm{~b})$ & $180.1 \pm 1.5(\mathrm{~b})$ & $177.6 \pm 1.6(\mathrm{c})$ & $-0.897^{* *}$ \\
HD_15_ZZ & $190.2 \pm 3.5(\mathrm{a})$ & $187.9 \pm 2.3(\mathrm{~b})$ & $187.6 \pm 1.8(\mathrm{bc})$ & $186.8 \pm 1.8(\mathrm{bc})$ & $186.4 \pm 1.9(\mathrm{c})$ & $183.1 \pm 2.2(\mathrm{~d})$ & $-0.938^{* *}$ \\
HD_average & $180.3 \pm 11.4(\mathrm{a})$ & $179.1 \pm 10.7(\mathrm{a})$ & $178.8 \pm 10.7(\mathrm{a})$ & $178.4 \pm 10.4(\mathrm{ab})$ & $178.3 \pm 10.3(\mathrm{ab})$ & $176.0 \pm 9.5(\mathrm{~b})$ & $-0.915^{* *}$ \\
FD_14_SQ & $193.7 \pm 1.8(\mathrm{a})$ & $192.2 \pm 1.8(\mathrm{~b})$ & $191.4 \pm 2.0(\mathrm{~b})$ & $191.5 \pm 1.5(\mathrm{~b})$ & $191.9 \pm 2.6(\mathrm{~b})$ & $188.3 \pm 2.5(\mathrm{c})$ & $-0.838^{*}$ \\
FD_15_SQ & $198.4 \pm 1.0(\mathrm{a})$ & $197.5 \pm 1.0(\mathrm{~b})$ & $197.1 \pm 0.8(\mathrm{~b})$ & $197.4 \pm 0.7(\mathrm{~b})$ & $196.9 \pm 1.0(\mathrm{~b})$ & $194.8 \pm 1.4(\mathrm{c})$ & $-0.867^{*}$ \\
FD_14_ZMD & $181.3 \pm 1.0(\mathrm{a})$ & $179.5 \pm 1.8(\mathrm{~b})$ & $179.2 \pm 1.8(\mathrm{~b})$ & $179.5 \pm 1.8(\mathrm{~b})$ & $178.7 \pm 1.9(\mathrm{~b})$ & $177.0 \pm 1.8(\mathrm{c})$ & $-0.907^{* *}$ \\
FD_15_ZMD & $167.4 \pm 2.1(\mathrm{a})$ & $166.5 \pm 1.5(\mathrm{ab})$ & $166.1 \pm 1.4(\mathrm{~b})$ & $165.9 \pm 1.4(\mathrm{~b})$ & $166.4 \pm 1.6(\mathrm{ab})$ & $165.6 \pm 1.6(\mathrm{~b})$ & $-0.813^{*}$ \\
FD_14_ZZ & $190.6 \pm 1.6(\mathrm{a})$ & $189.5 \pm 1.4(\mathrm{ab})$ & $189.0 \pm 1.5(\mathrm{~b})$ & $188.8 \pm 1.3(\mathrm{~b})$ & $188.6 \pm 1.3(\mathrm{~b})$ & $185.4 \pm 1.9(\mathrm{c})$ & $-0.885^{* *}$ \\
FD_15_ZZ & $199.3 \pm 1.7(\mathrm{a})$ & $198.2 \pm 1.6(\mathrm{ab})$ & $197.9 \pm 1.6(\mathrm{bc})$ & $197.3 \pm 1.6(\mathrm{bc})$ & $196.8 \pm 1.6(\mathrm{c})$ & $192.9 \pm 2.6(\mathrm{~d})$ & $-0.889^{* *}$ \\
FD_average & $189.1 \pm 11.4(\mathrm{a})$ & $188.1 \pm 11.2(\mathrm{a})$ & $187.6 \pm 11.3(\mathrm{ab})$ & $187.6 \pm 11.1(\mathrm{ab})$ & $187.4 \pm 11.0(\mathrm{ab})$ & $184.9 \pm 10.2(\mathrm{~b})$ & $-0.886^{* *}$ \\
\hline
\end{tabular}

Lowercase letters indicate significant differences at the 0.05 level; * and ${ }^{* *}$ indicates significant differences at the 0.05 and 0.01 level, respectively. The correlation coefficients $(r)$ were calculated between the mean values of each grade and corresponding rank $\left(r_{0.05,5}=0.754\right.$ and $\left.r_{0.01,5}=0.875\right)$; the ranks of the growth habit were calculated via the arithmetic means obtained during a two-year period, and all the data of all discrepant individuals were omitted

For Ppd-D1, the haplotypes identified among the materials were divided into three types in accordance with the reports of Guo et al. (2010) [31] and Chen et al. (2013) [13]: Hapl I (34.85\%, sensitive), Hapl II (0.5\%, Chinese Spring, insensitive), and Hapl VII (64.6\%, sensitive). Only one variety (Chinese Spring) had a $2.0-\mathrm{kb}$ deletion in the promoter region and thus should be designated Ppd-D1a (insensitive, theoretical relatively short HD and FD). Thus, Hapl I and Hapl VII could be considered recessive alleles in this study (Table 6).

\section{Effects of single Ppd-1 alleles on HD and FD}

One hundred and twenty-five materials that had double-recessive $V r n$ alleles $(v r n-B 1+v r n-D 1)$ were selected to evaluate the influence of $P p d-B 1$ or $P p d-D 1$ on plant traits. There were four genotypes, but no significant differences were found among groups according to
ANOVA results. Because inconsistencies between the ANOVA and LSR test results were sometimes detected, multiple comparisons were subsequently performed. The average values for phenotypes among the four groups differed significantly for only five traits (three traits in Zhumadian). Furthermore, varieties that harboured "Sonora 64 type" ("N: N: S") showed the shortest HD and FD. This result was consistent with those reported by Díaz et al. (2012) [28] (Table 7).

Regarding $P p d-D 1$, two genotypes were found, and no significant differences were detected via the LSR method between groups. We predicted that two recessive types of alleles did not contribute to the advancement of heading and flowering time. With respect to the comparisons of the mean values of $P p d-B 1$ and $P p d-D 1$ on $\mathrm{HD}$ and FD, the effects of $P p d-B 1$ were somewhat stronger than those of Ppd-D1 (Table 7).
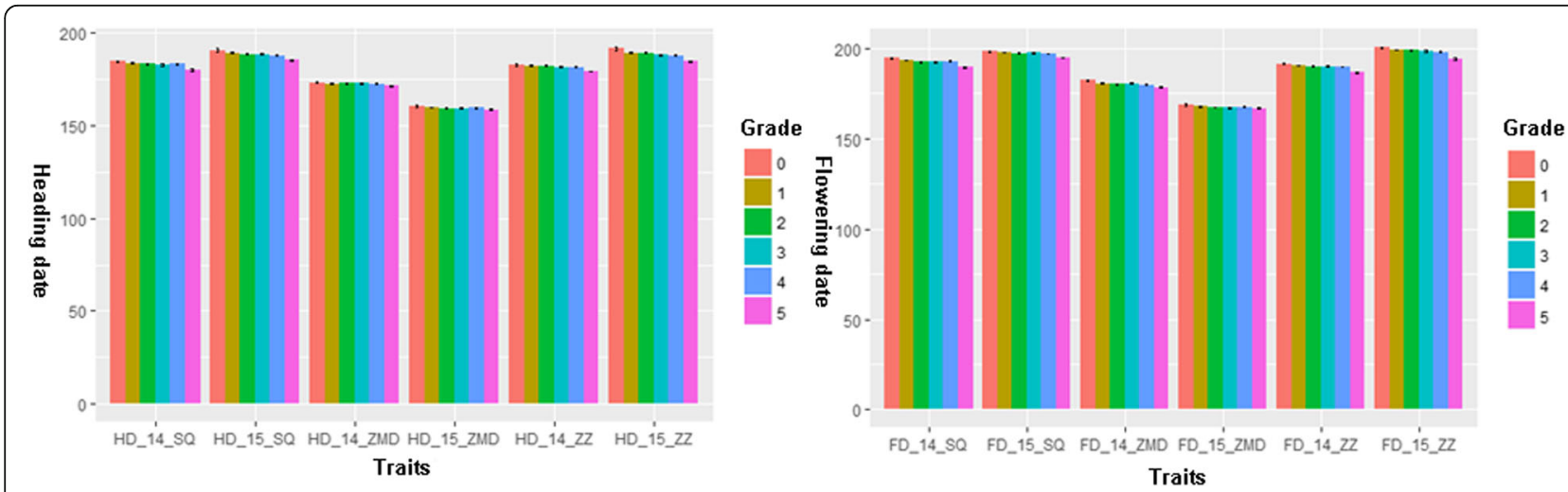

Fig. 2 Correlations between growth habits and heading and flowering date (HD and FD) in six environments 
Table 3 Distribution rates of $\mathrm{Vrn}-1$ and their consistency with results of identification of W/S growth habit

\begin{tabular}{lllllll}
\hline Genotype & Material number (only) & Frequency (\%) & Speculation of winter/spring habit & Winter & Spring & Consistency (\%) \\
\hline Vrn-B1a (only) & 5 & 2.5 & Spring & 0 & 5 & 100.0 \\
Vrn-B1b (only) & 4 & 2.0 & Spring & 0 & 4 & 100.0 \\
Vrn-D1a (only) & 47 & 23.7 & Spring & 24 & 23 & 48.9 \\
Vrn-D1b (only) & 16 & 8.1 & Spring & 12 & 4 & 25.0 \\
Vrn-B1b + Vrn-D1a & 1 & 0.5 & Spring & 0 & 1 & 100.0 \\
Vrn-B1+ vrn-D1 & 125 & 63.1 & Winter & $119 / 117$ & $4 / 6$ & $96.8 / 95.2$ \\
Total & 198 & 100.0 & & $155 / 153$ & $41 / 43$ & $79.8 / 78.2$ \\
\hline
\end{tabular}

The values in parentheses represent the percentage in each group; "only" in parentheses indicates that the value is specific to the single genotype

\section{Interactive effects of Ppd-1 combinations on HD and FD}

We also examined and assessed the interactive effects of Ppd-B1 and Ppd-D1 combinations. Similarly, no significant differences were found according to the ANOVA results. A total of eight genotypes that contained two Ppd alleles were surveyed, in which the type "N: N: S + Hapl VII" constituted the largest proportion (29, 23.6\%), while the percentages of "T: I: N + Hapl I or Hapl VII" were the lowest (Table 8). With respect to their effects, the LSR method revealed significant differences in the mean values among groups for four traits (three in Zhumadian and one in Zhengzhou). We suspected that $P p d-1$, especially $P p d-B 1$, functioned only in specific environments. Generally, the mean values of HD and FD were advanced by the $P p d-1$ combinations by only $0.2-0.5$ d. Therefore, the effects of Ppd-1 combinations were stronger than those of single $P p d-B 1$ or $P p d-D 1$ alleles but were far weaker than those of $\mathrm{Vrn}$ alleles (5-7 d earlier heading or flowering in Zhengzhou and Zhumadian). With respect to the individual genotypes, the rank order of their effects on growth period was as follows: Ppd-1>Ppd-B1 $>$ Ppd-D1.

\section{Discussion}

\section{Consistency of marker analysis and growth habit identification}

Wheat is the major crop in the YHW in terms of yield and area in China. This region is located in the transition zone of winter and spring wheat cultivation, where semi-winter varieties and weak spring cultivars are also planted. However, inconsistencies sometimes occur between registered and empirical results. Hence, the precise identification of winter/spring growth habits for newly registered varieties is necessary and helpful not only for the rational use of varieties but also for the provision of vital information for breeders in the YHW. Here, growth habits were examined during a two-year period via a novel field spring sowing identification method and materials along with marker-assisted selection (MAS).

We believe that our identification method in the field is more practical than that conducted in greenhouses, where the materials are grown under conditions closely related to those in the field. Furthermore, correlation analysis revealed that the phenotypic data during two

Table 4 LSR method of multiple comparison for the effects of Vrn-B1 combined with Vrn-D1

\begin{tabular}{|c|c|c|c|c|c|c|c|c|}
\hline Types & $\mathrm{N}$ & HD_14_SQ & HD_15_SQ & HD_14_ZMD & HD_15_ZMD & HD_14_ZZ & HD_15_ZZ & HD_average \\
\hline$v r n-B 1+v r n-D 1$ & 125 & $183.1 \pm 2.3(\mathrm{a})$ & $188.5 \pm 2.2(a)$ & $172.2 \pm 1.6(a)$ & $159.1 \pm 1.3(a)$ & $181.8 \pm 1.3(\mathrm{a})$ & $188.7 \pm 2.5(a)$ & $178.9 \pm 10.7(a)$ \\
\hline Vrn-B1a + vrn-D1 & 5 & $179.1 \pm 0.8(b c)$ & $184.6 \pm 0.8(b c)$ & $171.2 \pm 0.4(a b)$ & $157.8 \pm 0.5(a b)$ & $178.6 \pm 0.8(b c)$ & $184.4 \pm 1.6(\mathrm{bc})$ & $175.9 \pm 9.5(\mathrm{a})$ \\
\hline Vrn-B1b + vrn-D1 & 4 & $177.5 \pm 1.9(c)$ & $182.5 \pm 0.7(c)$ & $170.0 \pm 1.1(b)$ & $156.7 \pm 2.0(b)$ & $177.3 \pm 1.1(\mathrm{c})$ & $181.7 \pm 1.6(c)$ & $174.3 \pm 9.1(a)$ \\
\hline$v r n-B 1+V r n-D 1 a$ & 47 & $181.2 \pm 3.2(a b)$ & $186.5 \pm 2.6(\mathrm{ab})$ & $171.5 \pm 1.3(\mathrm{ab})$ & $158.8 \pm 1.4(a)$ & $179.8 \pm 1.8(a b)$ & $186.1 \pm 2.6(a b)$ & $177.4 \pm 10.0(a)$ \\
\hline$V r n-B 1 b+V r n-D 1 a$ & 1 & $180.5 \pm N A(a b c)$ & $184.0 \pm N A(b c)$ & $171.0 \pm N A(a b)$ & $159.0 \pm N A(a)$ & $178.5 \pm N A(b c)$ & $184.5 \pm N A(b c)$ & $176.3 \pm 9.8(a)$ \\
\hline$v r n-B 1+V r n-D 1 b$ & 16 & $180.8 \pm 2.5(a b c)$ & $186.5 \pm 2.7(a b)$ & $171.5 \pm 1.4(a b)$ & $158.7 \pm 1.1(a b)$ & $179.7 \pm 1.9(a b c)$ & $186.75 \pm 2.9(a b)$ & $177.4 \pm 10.8(a)$ \\
\hline Types & N & FD_14_SQ & FD_15_SQ & FD_14_ZMD & FD_15_ZMD & FD_14_ZZ & FD_15_ZZ & FD_average \\
\hline$v r n-B 1+v r n-D 1$ & 125 & $192.9 \pm 1.9(\mathrm{a})$ & $197.4 \pm 1.0(a)$ & $180.4 \pm 1.9(a)$ & $167.4 \pm 1.7(a)$ & $190.4 \pm 1.5(\mathrm{a})$ & $198.9 \pm 1.8(a)$ & $187.9 \pm 11.1(\mathrm{a})$ \\
\hline Vrn-B1a + vrn-D1 & 5 & $190.4 \pm 0.8(a b)$ & $195.0 \pm 0.6(b c)$ & $181.2 \pm 1.1(\mathrm{a})$ & $165.8 \pm 1.0(a)$ & $187.0 \pm 0.9(b c)$ & $195.0 \pm 1.5(b c)$ & $185.7 \pm 10.3(a)$ \\
\hline Vrn-B1b + vrn-D1 & 4 & $187.1 \pm 0.9(b)$ & $193.5 \pm 0.7(c)$ & $177.1 \pm 1.2(b)$ & $165.3 \pm 1.3(a)$ & $184.7 \pm 1.5(\mathrm{c})$ & $191.3 \pm 2.2(d)$ & $183.2 \pm 9.8(a)$ \\
\hline$v r n-B 1+V r n-D 1 a$ & 47 & $191.0 \pm 3.23(a)$ & $195.9 \pm 1.6(\mathrm{ab})$ & $178.7 \pm 1.9(\mathrm{ab})$ & $167.2 \pm 1.5(\mathrm{a})$ & $187.9 \pm 2.1(a b)$ & $196.2 \pm 3.0(a b c)$ & $186.2 \pm 10.7(a)$ \\
\hline$V r n-B 1 b+V r n-D 1 a$ & 1 & $190.0 \pm N A(a b)$ & $195.0 \pm N A(b c)$ & $181.0 \pm N A(a)$ & $167.5 \pm \mathrm{NA}(\mathrm{a})$ & $187.0 \pm N A(b c)$ & $193.0 \pm N A(c d)$ & $185.6 \pm 10.1(a)$ \\
\hline$v r n-B 1+V r n-D 1 b$ & 16 & $191.6 \pm 3.3(a)$ & $196.1 \pm 1.6(a b)$ & $178.6 \pm 2.1(a b)$ & $167 \pm 1.1(\mathrm{a})$ & $187.7 \pm 2.5(a b)$ & $196.7 \pm 2.9(a b)$ & $186.4 \pm 11.5(\mathrm{a})$ \\
\hline
\end{tabular}

Letters in parentheses indicate a significant difference at the 0.05 level; decimal values preceded by " \pm " indicate standard deviation 
Table 5 Allelic variants of $P p d-B 1$

\begin{tabular}{|c|c|c|c|c|c|}
\hline Genotype & Sonora 64 type & Intact CS type & Truncated CS type & $N$ & Proportion (\%) \\
\hline \multirow[t]{6}{*}{$P p d-B 1$} & No & No & No & 61 & 30.81 \\
\hline & Yes & No & No & 68 & 34.34 \\
\hline & No & Yes & No & 2 & 1.01 \\
\hline & No & No & Yes & 50 & 25.25 \\
\hline & Yes & No & Yes & 2 & 1.01 \\
\hline & No & Yes & Yes & 15 & 7.58 \\
\hline Total & $35.3 \%$ & $8.6 \%$ & $33.8 \%$ & 198 & 100 \\
\hline
\end{tabular}

years were also consistent between years. In comparison with registered information, 10 of 155 (6.45\%, 2016) winter wheat varieties were inconsistent (Zhongchuang805, Taikong6, Xiaoyan22, etc.), and similar situations were observed in other groups-in particular, 43 spring wheat varieties containing $13(25.6 \%, 2016)$ inconsistent samples (Bainong3217, Luohan6, Xinong979, etc.). We doubted that the reason for this situation was due to their early registration before the materials were rigorously identified. In total, the consistency was approximately $90 \%$, although some varieties presented discrepancies, and our method was more convenient than the report of Gardener and Barnett [32].

More vital clues that we wanted to examine included the consistency between vernalization alleles and growth habit. The results indicated that all ten Vrn-B1 genotypes of spring wheat varieties presented a value of $100 \%$, whereas Vrn-D1 exhibited lower results. Among the 43 cultivars ranked as grade 5 (data from2016, Xinzheng), $37(86.04 \%)$ carried at least one of the tested dominant vernalization alleles and were classified as spring varieties; the other 6 varieties carried the recessive alleles at the three vernalization loci. For winter types, 131 of 155 (84.51\%) accessions presented similar consistency. We predicted that there are two main factors that could be responsible for this phenomenon. First, a single individual is genotyped, whereas the phenotype is assessed on a plot scale of multiple individuals, and there may be some variation among individual seeds. Second, two other major pathways also control heading and flowering dates in plants, i.e., the phytohormone gibberellic acid (GA) and the autonomous pathways, in addition to the vernalization and photoperiod pathways [33, 34].

Allelic distributions of $V r n-1$ revealed trends and orientations in the YHW.

As no dominant allele of $V r n-A 1$ or $V r n-B 3$ was detected, which are probably the two genes that have the strongest effects of those examined, the allelic distributions of the dominant $V r n-B 1$ and $V r n-D 1$ alleles are likely responsible for the spring genotypes of wheat varieties. Indeed, the scarcity and decreasing frequency of the Vrn-A1 and Vrn-B3 loci in the YHW have been previously discussed by Zhang et al. (2008) and Chen et al. (2013) [4, 13]. We suspected that the frequencies of recessive $v r n-B 1$ and $v r n-D 1$ likely increased via direct selection due to their contributions to yield traits because of the long maturation period. This inference was in agreement with that reported in the literature [14, 35]. Furthermore, the frequency of dominant Vrn-D1 was higher than that of $V r n-B 1$ in our tested materials. These results were also consistent with the previous report of Zhang et al. (2015) [36].

Although only 10 dominant Vrn-B1 alleles (5.0\%) were discovered in the spring genotypes, the consistency of the marker-growth habit $(100 \%)$ was better than that of $V r n-D 1$. Additionally, we found that accessions with single $V r n-B 1 b$ alleles exhibited the earliest HD and FD in the six environments; these effects were stronger than those for Vrn-B1a, Vrn-D1b and Vrn-D1a. These results were consistent with those of previous studies in which the rank order was $V r n-A 1>V r n-B 1>V r n-D 1$ [37]. Santra et al. (2009) reported that a novel $V r n-B 1 b$ allele that resulted from a $36-\mathrm{bp}$ deletion within intron 1 , which is

Table 6 Allelic variants of Ppd-D1

\begin{tabular}{|c|c|c|c|c|c|c|}
\hline Genotype & 2-kb deletion & Transposable element (TE)insertion & 5-bp deletion & 16-bp insertion & Number & Proportion (\%) \\
\hline \multirow[t]{3}{*}{ Ppd-D1 } & Hapl I & & & & 69 & 34.85 \\
\hline & Hapl II & & & & 1 & 0.51 \\
\hline & Hap/ VII & & & & 128 & 64.65 \\
\hline Total & & & & & 198 & 100 \\
\hline
\end{tabular}

The method for dissecting the haplotypes of Ppd-D1was described by Guo et al. (2010) 
Table 7 The effects of single Ppd-B1 and Ppd-D1alleles on HD and FD

\begin{tabular}{|c|c|c|c|c|c|c|c|c|}
\hline Type & $N$ & HD_14_SQ & HD_15_SQ & HD_14_ZMD & HD_15_ZMD & HD_14_ZZ & HD_15_ZZ & HD_average \\
\hline$N: N: N$ & 39 & $183.2 \pm 2.7(a b)$ & $188.7 \pm 2.6(\mathrm{a})$ & $172.1 \pm 1.5(b)$ & $159.2 \pm 1.4(b)$ & $181.8 \pm 1.5(\mathrm{ab})$ & $189.2 \pm 2.6(\mathrm{a})$ & $179.1 \pm 10.9(a)$ \\
\hline$T: N: N$ & 30 & $183.3 \pm 2.1(a b)$ & $188.6 \pm 1.9(a)$ & $172.7 \pm 1.7(a b)$ & $159.2 \pm 1.2(b)$ & $181.9 \pm 1.4(a b)$ & $188.4 \pm 2.6(a)$ & $179.0 \pm 10.6(a)$ \\
\hline$T: I: N$ & 10 & $184.1 \pm 2.1(\mathrm{a})$ & $189.3 \pm 1.9(\mathrm{a})$ & $173.6 \pm 1.3(\mathrm{a})$ & $160.2 \pm 0.8(a)$ & $182.7 \pm 0.7(a)$ & $189.7 \pm 1.3(\mathrm{a})$ & $179.9 \pm 10.5(\mathrm{a})$ \\
\hline$N: N: S$ & 44 & $182.6 \pm 2.1(b)$ & $188.2 \pm 2.1(\mathrm{a})$ & $171.8 \pm 1.4(b)$ & $158.8 \pm 1.3(b)$ & $181.7 \pm 1.3(b)$ & $188.2 \pm 2.5(\mathrm{a})$ & $178.6 \pm 10.6(a)$ \\
\hline Type & $N$ & FD_14_SQ & FD_15_SQ & FD_14_ZMD & FD_15_ZMD & FD_14_ZZ & FD_15_ZZ & FD_average \\
\hline$N: N: N$ & 39 & $193.3 \pm 2.5(\mathrm{a})$ & $197.3 \pm 1.3(\mathrm{a})$ & $180.4 \pm 2.1(\mathrm{a})$ & $167.5 \pm 1.9(a b)$ & $190.5 \pm 1.8(\mathrm{a})$ & $199.2 \pm 2.1(\mathrm{a})$ & $188.1 \pm 11.3(\mathrm{a})$ \\
\hline$T: N: N$ & 30 & $192.7 \pm 1.6(\mathrm{a})$ & $197.5 \pm 0.9(\mathrm{a})$ & $180.5 \pm 1.9$ (a) & $167.5 \pm 1.4(b)$ & $190.2 \pm 1.4(\mathrm{a})$ & $198.7 \pm 2.2(\mathrm{a})$ & $187.9 \pm 11.1(\mathrm{a})$ \\
\hline$T: I: N$ & 10 & $193.1 \pm 0.9(\mathrm{a})$ & $197.7 \pm 0.8(a)$ & $181.0 \pm 1.4(\mathrm{a})$ & $168.5 \pm 1.4(a)$ & $190.9 \pm 0.8(\mathrm{a})$ & $199.4 \pm 0.8(a)$ & $188.5 \pm 10.8(a)$ \\
\hline$N: N: S$ & 44 & $192.7 \pm 1.5(\mathrm{a})$ & $197.3 \pm 0.8(\mathrm{a})$ & $180.3 \pm 1.8(\mathrm{a})$ & $166.9 \pm 1.6(b)$ & $190.3 \pm 1.4(\mathrm{a})$ & $198.7 \pm 1.5(\mathrm{a})$ & $187.7 \pm 11.2(\mathrm{a})$ \\
\hline Type & N & HD_14_SQ & HD_15_SQ & HD_14_ZMD & HD_15_ZMD & HD_14_ZZ & HD_15_ZZ & HD_average \\
\hline Hapl I & 45 & $183.1 \pm 2.2(\mathrm{a})$ & $188.5 \pm 2.0(\mathrm{a})$ & $172.3 \pm 1.5(\mathrm{a})$ & $159.1 \pm 1.1(\mathrm{a})$ & $181.9 \pm 1.3(\mathrm{a})$ & $188.8 \pm 2.2(\mathrm{a})$ & $179.0 \pm 10.6(a)$ \\
\hline Hap/ VII & 77 & $183.1 \pm 2.4(\mathrm{a})$ & $188.5 \pm 2.3(\mathrm{a})$ & $172.2 \pm 1.6(\mathrm{a})$ & $159.1 \pm 1.4(a)$ & $181.8 \pm 1.4(\mathrm{a})$ & $188.6 \pm 2.6(a)$ & $178.9 \pm 10.7(a)$ \\
\hline Type & $N$ & FD_14_SQ & FD_15_SQ & FD_14_ZMD & FD_15_ZMD & FD_14_ZZ & FD_15_ZZ & FD_average \\
\hline HaplI & 45 & $192.7 \pm 1.5(\mathrm{a})$ & $197.3 \pm 1.0(a)$ & $180.7 \pm 1.7(a)$ & $167.2 \pm 1.4(a)$ & $190.4 \pm 1.4(\mathrm{a})$ & $199.1 \pm 1.7(\mathrm{a})$ & $187.9 \pm 11.1(a)$ \\
\hline Hap/ VII & 77 & $193.1 \pm 2.1$ (a) & $197.4 \pm 1.1$ (a) & $180.3 \pm 2.0(\mathrm{a})$ & $167.4 \pm 1.8(\mathrm{a})$ & $190.4 \pm 1.5(\mathrm{a})$ & $198.8 \pm 1.9(\mathrm{a})$ & $187.9 \pm 11.2(\mathrm{a})$ \\
\hline
\end{tabular}

"N" in the "Type" column indicates that no target bands were amplified in the "Truncated CS type (425 bp)", "Intact CS type (994 bp)"or"Sonora64 (223 bp)"CNVs at the Ppd-B1 locus

referred to as 'Alpowa' and carries the winter growth habit alleles vrn-A1 and vrn-B1, was likely to cause a spring growth habit; however, the authors did not provide sufficient evidence [14].

The dominant Vrn-D1 locus occurs in the most popular types and is distributed throughout nearly the entire wheat production region $[4,13]$. The previous data also established that carriers of $V r n-A 1$ or $V r n-D 1$ tend to produce longer spikes than do carriers of $V r n-B 1$. As a result, the $V r n-D 1$ genotypes were prevalent in China $[38,39]$. In the present study, in terms of $V r n-D 1$, the allelic frequency reached $31.8 \%$, which represented the most dominant allele distribution in the population. Thus, the proportion was similar to previous reports; however, poor consistency in the growth habit of Zhengzhou was observed. Thus, it would be interesting to test whether Vrn-D1 and other alleles interact to influence growth habits and period (HD and FD).

The results of the $V r n-1$ combination analysis revealed that only one accession carried Vrn-B1b+Vrn-D1a alleles; thus, the samples were so limited that phenotypic data were not statistically representative. For genotypic analysis, in combination with growth habit identification, $63.1 \%$ of the materials that had three recessive alleles all belonged to winter or semi-winter wheat (grade 0-4). This tendency was in accordance with that reported by Sun et al. (2009, 61.1\%). Moreover, the ANOVA and LSR tests revealed that the mean values of the HD, FD and growth habit among six genotypes differed significantly, which also indicated that the Vrn-1 combinations were tightly associated with phenotypes. Although the effects of these combinations on HD and FD were not definitively stronger than those of single Vrn-B1 alleles, the growth habit level was divided in greater detail. This division will enable a more precise identification of vernalization requirements for accessions at molecular levels.

\section{Interactive effects detected between the Ppd-B1 and Ppd- D1 alleles}

Photoperiod responses are controlled by members of the pseudo-response regulator $(P R R)$ gene family in plants. In general, the potential of $P p d-1$ alleles to affect insensitivity has been ranked as $P p d-D 1>P p d-B 1>P p d-A 1$ [17, $38]$. However, in the present study, only one accession (Chinese spring) was found to carry a 415-bp band that indicated a genotype of Ppd-D1a. Thus, it was difficult to precisely evaluate its effect on phenotype statistically. According to the criterion of previous research, two sensitive haplotypes (Hapl I and Hapl II) of Ppd-DI were used for the evaluation for their distribution and effects $[13,31]$.

For $P p d-B 1$, we only examined the polymorphisms of CNVs of the Ppd-B1 locus because of its tight association with heading and flowering time. Indeed, Zhang et al. (2015) designated eight haplotypes according to the combinations of CNVs of Ppd-B1 and found that the cultivar with Ppd-B1_hapl-VI demonstrated the earliest heading and flowering times [36]. However, the results were not consistent with those of Díaz et al. (2012) [28]. In the present study, 125 accessions carrying recessive $v r n-B 1$ and $v r n-D 1$ alleles were selected. With respect to Ppd-B1, we found that wheat cultivars with "Sonora 64" $P p d-B 1 a$ alleles flower earlier than those with "Chinese 
$\mid \begin{array}{llllllllll} & 1 & 1\end{array}$

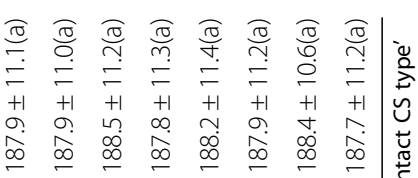

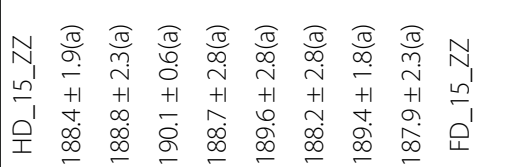

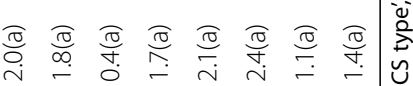

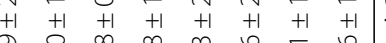

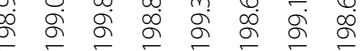

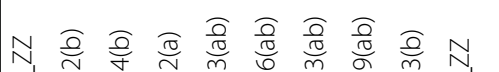

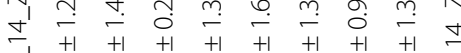

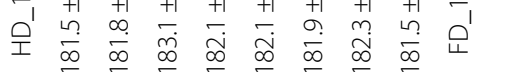

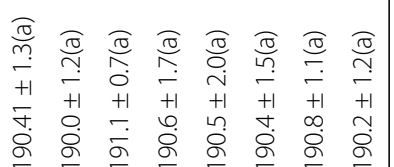

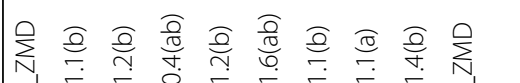

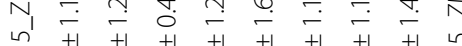

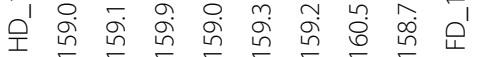

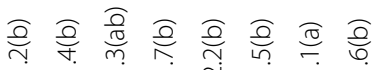

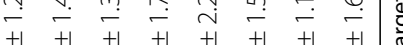

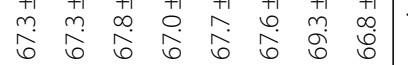

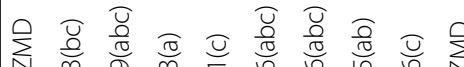

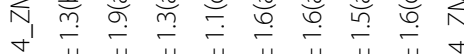

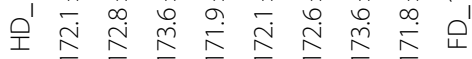

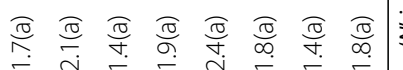

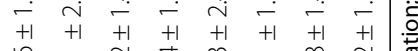

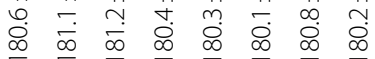

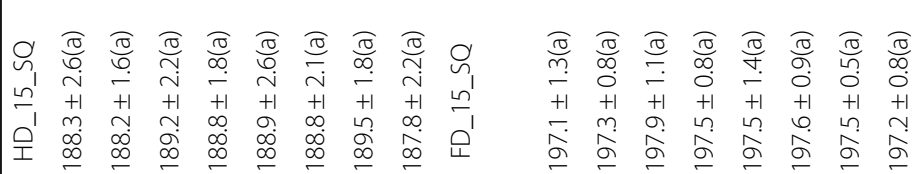

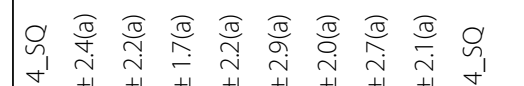

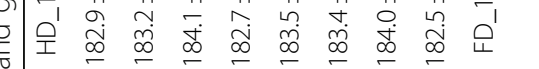

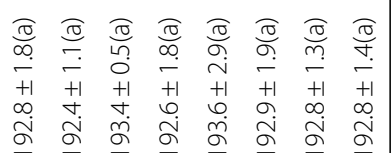

을

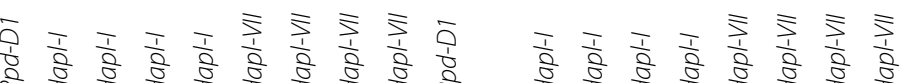

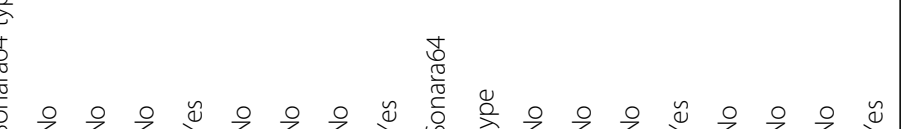


Spring" alleles, which was in accordance with Díaz et al. (2010). Furthermore, we found that six types of combinations emerged, and the "Truncated CS type" and "Intact CS type" did not simultaneously emerge for $P p d-B 1$. These results were also not the same as those reported by Chen et al. (2013). We suspected that the complex genetic background (genotypes mixed with $V r n-1$ genes) would hinder us from providing definitive results. Thus, we believed that our method was possibly more reliable than previous methods because of the uniform background [30]. With respect to Ppd-D1a, the rare diversity of the $P p d-D 1$ allele could not be used to exactly evaluate the effects of the variants, and no significant differences were observed between the two haplotypes (Hapl I and Hapl II).

\section{Comparison of effects on growth period (HD and FD) between the Vrn-1 and Ppd-1 alleles}

Moreover, from a comprehensive perspective, we concluded that, compared with Ppd-1, Vrn-1 played a major role in regulating heading and flowering traits as well as growth habit. At the Vrn-1 locus, cultivars with the $V r n-B 1 b+v r n-D 1$ (174.3 d for HD, $183.2 \mathrm{~d}$ for FD) allele both headed and flowered earlier by approximately 4 days than did cultivars with the vrn-B1 + vrn-D1 (178.9 $\mathrm{d}$ for HD, $187.9 \mathrm{~d}$ for FD) allele (Table 4). Whereas at the Ppd-1 locus, cultivars with the "N: N: S" allele combination (178.6 d for HD, $187.7 \mathrm{~d}$ for FD) both headed and flowered approximately 1 day earlier than did cultivars with the "T: $I: N$ " allele combination $(179.9 \mathrm{~d}$ for HD, $188.5 \mathrm{~d}$ for FD) (Table 7). Indeed, the interactive effects of $V r n-1$ and $P p d-1$ gene combinations were also detected in our research. However, the results of ANOVA and LSR tests revealed weak interactions between Vrn-B1 and Ppd-B1, Vrn-D1 and Ppd-D1, Vrn-B1and Ppd-D1, Vrn-Dland Ppd-B1 (data not shown). We suspected that the complex genetic background in natural populations would make it difficult to reveal this interaction. In a previous study, Shcherban et al. (2014) also found that the haplotypes Ppd-D1al Vrn-B1a or Ppd-D1a/Vrn-B1a did not differ significantly in heading time from the respective Vrn-1haplotypesharbouring the sensitive allele Ppd-D1b [40]. This finding suggests that it is better for us to examine the interaction between $P p d-1$ and $V r n-1$ in biparental populations.

\section{Conclusion}

In the present study, we dissected the Vrn-1 and Ppd-1 gene composition and found that $V r n-1$, rather than Ppd-1, played a major role in controlling vernalization and photoperiod responses in this region. The work will be helpful for guiding the breeding of wheat in the Yellow and Huai wheat production region.

\section{Methods}

We tested 198 cultivars (lines) including historic varieties, commercial varieties, and newly bred varieties originating from the YHW. Among them, 159 accessions were from Henan, 10 accessions were from Shandong, 10 accessions were from Shaanxi, 8 accessions were from Jiangsu, 4 accessions were from Hebei, 4 accessions were from Beijing, 1 accession was from Shanxi, 1 accession was from Anhui, and 1 accession (Chinese Spring) was from Sichuan (Table 1). The entire original source of the plant materials used in our study was kindly provided by other labs. We complied with the Convention on the Trade in Endangered Species of Wild Fauna and Flora: https://www.cites.org.

\section{Characterization of winter/spring growth habits}

Although the growth habit for assessing vernalization is already well established, identification of the exact materials involved is necessary because of differences in environmental conditions. The tested materials were planted at the Zhengzhou Scientific Research and Education Center of Henan Agriculture University $\left(113.7^{\circ} \mathrm{E}\right.$, $34.7^{\circ} \mathrm{N}$ ) on 12 March 2015 and at another test site [ZhengHan Seed Technology Co. Ltd., XinZheng (113.7 E, $\left.34.4^{\circ} \mathrm{N}\right)$ ] on 12 March 2016. Seeds were sown in $1.0-\mathrm{m}$ rows, and individual seeds were spaced $6.67 \mathrm{~cm}$ apart; 15 seedlings were reserved per row after wheat seedling emergence. Two replications were planted for reliable data collection. The stage of maturity and percentage of headed spikes were recorded on 25 June in the same year; we repeated these measurements one week later. The growth habit of the materials was divided into grades numbered 0 to 5 . The criteria were as follows: 0 , no jointing and booting; 1 , partial main stem headed; 2, main stem and a few tillers headed; 3 , normal heading but abnormal grain filling and immature; 4, normal heading and grain filling but premature; 5 , normal maturity.

\section{Identification of $\mathrm{HD}$ and $\mathrm{FD}$}

The varieties used to assess agronomic traits were planted on 9 October 2013 and 2014 in Zhengzhou $\left(113.7^{\circ} \mathrm{E}, 34.7^{\circ} \mathrm{N}\right)$, on 15 October 2013 and 17 October 2014 in Shangqiu $\left(115.7^{\circ} \mathrm{E}, 34.5^{\circ} \mathrm{N}\right)$, and on 19 October 2013 and 5 November 2014 in Zhumadian $\left(114.0^{\circ} \mathrm{E}\right.$, $32.9^{\circ} \mathrm{N}$ ). All of these locations differed significantly in day length and climatic factors in Henan Province. Each material was planted in two $1.5-\mathrm{m}$ rows; there were 110 seeds per row, and the rows were spaced $23 \mathrm{~cm}$ apart. Two replications were planted at each location. Field management practices during our experiments were in accordance with agronomic practices commonly used in the area. The HD and FD were assessed on a plot scale of multiple individuals when more than half of the 
individual seedlings exhibited classic morphological traits for these events.

\section{DNA extraction and diagnostic markers for $V r n-1$ and Ppd-1}

DNA was extracted from the seedlings in accordance with a modified SDS-phenol-chloroform method [27]. The primers used were based on those described in many previous reports and were synthesized by Sangon Biotech Co., Ltd. (Shanghai) (Additional file 2: Table S1). To recognize the segments amplified from Ppd-B1, accessions harbouring $994 \mathrm{bp}, 425 \mathrm{bp}$, and $223 \mathrm{bp}$ were designated intact Chinese Spring type (I), truncated Chinese Spring type $(\mathrm{T})$, and Sonora 64 type $(\mathrm{S})$, respectively. If no bands were amplified in the materials, the genotypes were referred to as null (N) [28].

\section{PCR amplification and electrophoresis}

PCR amplification reactions were conducted in a $12-\mu \mathrm{L}$ reaction flask containing $40 \mathrm{ng}$ of genomic DNA, each primer at $2.5 \mu \mathrm{M}$, each $\mathrm{dNTP}$ at $200 \mu \mathrm{M}, 1 \times$ buffer containing1.5 $\mu \mathrm{M} \mathrm{MgCl}_{2}$, and 0.5 units of Taq polymerase. We used a Bio-Rad thermocycler with the following PCR conditions: $94{ }^{\circ} \mathrm{C}$ for $3 \mathrm{~min}$; 34 cycles of $94{ }^{\circ} \mathrm{C}$ for $30 \mathrm{~s}, 50^{\circ} \mathrm{C}$ to $65^{\circ} \mathrm{C}$ for $30 \mathrm{~s}$ (annealing temperatures for each primer pair are listed in Additional file 2: Table S1), and $72^{\circ} \mathrm{C}$ for $1 \mathrm{~min}$; and a final 10 -min extension at $72^{\circ}$ $\mathrm{C}$ for preservation. The PCR products were separated by electrophoresis either on a $0.8-1.2 \%$ agarose gel stained with ethidium bromide (EB) or an $8 \%$ nondenaturing polyacrylamide gel and visualized with silver staining [29].

\section{Statistical analysis}

A consistency index (\%) was used; this index indicated the number of accessions that originated from the consistent results of both genotype and field identifications divided by the total number of materials. The phenotypic data were imported into $R$ software ( $R$ 3.4.1) for analysis via ANOVA, Student's t-tests and correlation; we used the "reshape" and "agricolae" packages to perform these analyses and the "ggplot2" package for graphical construction.

\section{Additional file}

Additional file 1: Table S2. The genotypes of Ppd-1 and their effects on heading and flowering dates. (XLS $25 \mathrm{~kb}$ )

Additional file 2: Table S1. Primers used in this study. (XLS $71 \mathrm{~kb}$ )

\section{Abbreviations}

YHW: Yellow and Huai wheat production region.; HD: Heading date.; FD: Flowering date.; ZZ: Zhengzhou.; ZMD: Zhumadian.; SQ: Shangqiu.; ANOVA: Analysis of variance.; LSR: Least significant range method of multiple comparison.; CNVs: Copy number variations.; MAS: Marker-assisted selection.; PRR: Pseudo-response regulator.; WS: Winter/Spring growth habit.

\section{Acknowledgements}

The authors would like to thank Aimin Zhang for his support, advice and revisions regarding this manuscript as well as the Institute of Genetics and Developmental Biology, Chinese Academy of Sciences.

\section{Funding}

The design of the study and collection, analysis, and interpretation of data were supported by the National Basic Research Program of China

(2014CB138105); the molecular experiments were supported by the Youth Science and Technology Innovation Fund (30601440).

Availability of data and materials

All data are available in the additional files.

Authors' contributions

KZ conceived the project and prepared the trials. JW, GD and LC performed the experiments. SC analysed the data and was a major contributor in writing the manuscript. XC and HX performed statistical analysis and edited the manuscript. All authors read and approved the final manuscript.

Ethics approval and consent to participate

Not applicable.

Consent for publication

Not applicable

\section{Competing interests}

The authors declare that they have no competing interests.

\section{Publisher's Note}

Springer Nature remains neutral with regard to jurisdictional claims in published maps and institutional affiliations.

\section{Author details}

${ }^{1}$ College of Agronomy, Henan Agricultural University/Collaborative Innovation Center of Henan Grain Crops, Zhengzhou 450002, China. ${ }^{2}$ HuaGuan Seed Technology Co. Ltd., Zhoukou, Henan, China. ${ }^{3}$ YuLong Crops Research Institute, Xinzheng, Henan, China.

Received: 19 June 2018 Accepted: 3 December 2018

Published online: 27 December 2018

\section{References}

1. Trevaskis B, Hemming MN, Peacock WJ, Dennis ES. HVVRN2 responds to day length, whereas HVVRN1 is regulated by vernalization and developmental status. Plant Physiol. 2006:140:1397-405.

2. Yan L, Loukolanov A, Tranquilli G, Helguera M, Fahima T, Dubcovsky J. Positional cloning of the wheat vernalization gene VRN1. Proc Natl Acad Sci U S A. 2003;100:6263-8.

3. Gardner FP, Barnett RD. Vernalization of wheat cultivars and a triticale. Crop Sci. 1990:30:166-9.

4. Zhang XK, Xiao YG, Zhang Y, Xia XC, Dubcovsky J, He ZH. Allelic variation at vernalization genes $V r n-A 1, V r n-B 1, V r n-D 1$ and $V r n-B 3$ in Chinese common wheat cultivars and their association with growth habit. Crop Sci. 2008:48:458-70.

5. Distelfeld A, Li C, Dubcovsky J. Regulation of flowering in temperate cereals. Curr Opin Plant Biol. 2009:12:178-84.

6. Dubcovsky J, Lijavetzky D, Appendino L, Tranquilli G. Comparative RFLP mapping of Triticum monococcum genes controlling vernalization requirement. Theor Appl Genet. 1998;97:968-75

7. Iwaki K, Nishida J, Yanagisawa T, Yoshida H, Kato K. Genetic analysis of Vrn$B 1$ for vernalization requirement by using linked dCAPS markers in bread wheat (Triticum aestivum L.). Theor Appl Genet. 2002;104:571-6.

8. Levy YY, Mesnage S, Mylne JS, Gendall AR, Dean C. Multiple roles of ArabidopsisVRN1 in vernalization and flowering time control. Science. 2002:297:243-6.

9. Yan L, Helguera M, Kato K, Fukuyama S, Sherman J, Dubcovsky J. Allelic variation at the VRN-1 promoter region in polyploid wheat. Theor Appl Genet. 2004;109:1677-86. 
10. Fu D, Szücs P, Yan L, Helguera M, Skinner JS, Zitzewitz JV, et al. Large deletions within the first intron in VRN-1 are associated with spring growth habit in barley and wheat. Mol Gen Genomics. 2005;273:54-65.

11. Yan L, Fu D, Lin C, Blechl A, Tranquilli G, Bonafede M, et al. The wheat and barley vernalization gene VRN3 is an orthologue of FT. Proc Natl Acad Sci. 2006;103:19581-6.

12. Mclntosh RA, Yamazaki Y, Dubcovsky J, Rogers WJ, Morris C, Appels R, et al. Catalogue of gene symbols for wheat. In: Proceedings of 12th International wheat genetics Symposium, Yokohama, Japan.2013 http://www.shigen.nig. ac.jp/wheat/komugi/genes/macgene/2013/GeneCataloguelntroduction.pdf.

13. Chen F, Gao MX, Zhang JH, Zuo AH, Shang XL, Cui DQ. Molecular characterization of vernalization and response genes in bread wheat from the yellow and Huai Valley of China. BMC Plant Bio. 2013;13:199.

14. Santra DK, Santra M, Allan RE, Campbell KG, Kidwell KK. Genetic and molecular characterization of vernalization genes Vrn-A1, Vrn-B1, and Vrn-D1 in spring wheat germplasm from the Pacific northwest region of the U.S.a. Plant Breed. 2009;28:576-84.

15. Milec Z, Tomková L, Sumíková T, Pánková K. A new multiplex PCR test for the determination of $\mathrm{V} r n-B 1$ alleles in bread wheat (Triticum aestivum L.) Mol Breed. 2012;30:317-23.

16. Zhang J, Wang Y, Wu S, Yang JP, Liu H, Zhou Y. A single nucleotide polymorphism at the Vrn-D1 promoter region in common wheat is associated with vernalization response. Theor Appl Genet. 2012;125:1697-704.

17. Shcherban AB, Efremova TT, Salina EA. Identification of a new Vrn-B1 allele using two near-isogenic wheat lines with difference in heading time. Mol Breed. 2012;29:675-85.

18. Milec Z, Sumikova T, Tomkova L, Pánková K. Distribution of different Vrn-B1 alleles in hexaploid spring wheat germplasm. Euphytica. 2013;192:371-8.

19. Stelmakh AF. Genetic effects of Vrn genes on heading date and agronomic traits in bread wheat. Euphytica. 1993;65:53-60.

20. Chen A, Dubcovsky J. Wheat TILLING mutants show that the vernalization gene VRN1 down-regulates the flowering repressor VRN2 in leaves but is not essential for flowering. PLoS Genet. 2012;8:e1003134.

21. Danyluk J, Kane NA, Breton G, Limin AE, Fowler DB, Sarhan F. TaVRT-1, a putative transcription factor associated with vegetative to reproductive transition in cereals. Plant Physiol. 2003;132:1849-60.

22. Cockram J, Jones H, Leigh FJ, O'Sullivan D, Powell W, Laurie DA. Control of flowering time in temperate cereals: genes, domestication, and sustainable productivity. J Exp Bot. 2007:58:1231-44.

23. Foulkes MJ, Sylvester-Bradley R, Worland AJ, Snape JW. Effects of a photoperiod-response gene Ppd-D1 on yield potential and drought resistance in UK winter wheat. Euphytica. 2004;135:63-73.

24. Beales J, Turner A, Griffiths S, Snape JW, Laurie DA. A Pseudo-Response Regulator is misexpressed in the photoperiod insensitive Ppd-D1a mutant of wheat (Triticum aestivum L.). Theor Appl Genet. 2007;115:721-33.

25. Turner A, Beales J, Faure S, Dunford RP, Laurie DA. The pseudo-response regulator Ppd-H1 provides adaptation to photoperiod in barley. Science. 2005;310:1031-4.

26. Nishida H, Yoshida T, Kawakami K, Fujita M, Long B, Akashi Y, et al. Structural variation in the $5^{\prime}$ upstream region of photoperiod-insensitive alleles Ppd-A1 $a$ and Ppd-B1a identified in hexaploid wheat (Triticum aestivum L.), and their effect on heading time. Mol Breed. 2013;31:27-37.

27. Devos K, Gale MD. The use of random amplified polymorphic DNA markers in wheat. Theor Appl Genet. 1992;84:567-72.

28. Díaz A, Zikhali M, Turner AS, Isaac P, Laurie DA. Copy number variation affecting the photoperiod-B1 and vernalization-A1 genes is associated with altered flowering time in wheat (Triticum aestivum L.). PLoS One. 2012;7:e33234

29. Bassam BJ, Caetano-Anollés G, Gresshoff PM. Fast and sensitive silver staining of DNA in polyacrylamide gels. Anal Biochem. 1991;196:80-3.

30. Cane K, Eagles HA, Laurie DA, Trevaskis B, Vallance N, Eastwood RF, et al. Ppd-B1 and Ppd-D1 and their effects in southern Australian wheat. Crop Pasture Sci. 2013;64:100-14.

31. Guo ZA, Song YX, Zhou RH, Ren ZL, Jia JZ. Discovery, evaluation and distribution of haplotypes of the wheat Ppd-D1 gene. New Phytol. 2010; 185:841-51

32. Gardner FP, Barnett RD. Vernalization of wheat cultivars and atriticale. Crop Sci. 1990:30:166-9.

33. Yasuda S. Comparative studies on the development of spike primordia between cultivars of common wheat and barley. BerOhara Inst Landw Biol Okayama Univ. 1984;18:211-5.
34. Kato K, Yamagata $\mathrm{H}$. Method for evaluation of chilling requirement and narrow sense earliness of wheat cultivars. Jpn J Breed. 1988:38:172-86.

35. Sun QM, Zhou RH, Gao LF, Zhao GY, Jia JZ. The characterization and geographical distribution of the genes responsible for vernalization requirement in Chinese bread wheat. J Integr Plant Biol. 2009;51:423-32.

36. Zhang XF, Gao MX, Wang SS, Chen F, Cui DQ. Allelic variation at the vernalization and photoperiod sensitivity loci in Chinese winter wheat cultivars (Triticum aestivum L.). front. Plant Sci. 2015;6:470.

37. Loukoianov A, Yan L, Blechl A, Dubcovsky J. Regulation of VRNIvernalization genes in normal and transgenic polyploid wheat. Plant Physiol. 2005;138:2364-73.

38. Blake NK, Lanning SP, Martin JM, Doyle M, Sherman JD, et al. Effect of variation for major growth habit genes on maturity and yield in five spring wheat populations. Crop Sci. 2008;49:1211-20.

39. Trevaskis B, Bagnall DJ, Ellis MH, Peacock WJ, Dennis ES. MADS box genes control vernalization-induced flowering in cereals. Proc Natl Acad Sci U S A. 2003;100:13099-104.

40. Shcherban AB, Borner A, Salina EA. Effect of VRN-1 and PPD-D1 genes on heading time in European bread wheat cultivars. Plant Breed. 2015;134:49-55.

\section{Ready to submit your research? Choose BMC and benefit from:}

- fast, convenient online submission

- thorough peer review by experienced researchers in your field

- rapid publication on acceptance

- support for research data, including large and complex data types

- gold Open Access which fosters wider collaboration and increased citations

- maximum visibility for your research: over $100 \mathrm{M}$ website views per year

At $\mathrm{BMC}$, research is always in progress.

Learn more biomedcentral.com/submissions 